\title{
Selective Elimination of Axons Extended by Developing Cortical Neurons Is Dependent on Regional Locale: Experiments Utilizing Fetal Cortical Transplants
}

\author{
Dennis D. M. O'Leary' ${ }^{1}$ and Brent B. Stanfield ${ }^{2}$ \\ 'Departments of Neurology and Neurological Surgery and of Anatomy and Neurobiology, and The McDonnell Center for \\ Studies of Higher Brain Function, Washington University School of Medicine, St. Louis, Missouri 63110, and \\ ${ }^{2}$ National Institute of Mental Health, National Institutes of Health Animal Center, Poolesville, Maryland 20837
}

In adult rats, cortical neurons that extend an axon through the pyramidal tract (a major subcortical efferent projection of the neocortex) are limited to layer $V$ of about the rostral two-thirds of the neocortex. In neonates, however, pyramidal tract neurons are distributed throughout the neocortex, but all of those found in certain areas, such as the posterior occipital region (including primary visual cortex) selectively lose their pyramidal tract axon (Stanfield et al., 1982) yet maintain axon collaterals to other subcortical targets (O'Leary and Stanfield, 1985). To determine if the regional location of a developing pyramidal tract neuron critically influences the maintenance or elimination of the axon collaterals it initially extends, pieces of cortex from embryonic day 17 (E17) rat fetuses (exposed to ${ }^{3} \mathrm{H}$-thymidine on $\mathrm{E15}$ ) were transplanted heterotopically into the cortex of newborn (PO) rats; rostral cortex was placed into the posterior occipital region $(R \rightarrow O)$, or posterior occipital cortex into a rostral cortical locale $(O \rightarrow R)$. The retrograde tracers Fast blue (FB) and Diamidino yellow (DY) were used to assay for the presence of specific populations of cortical projection neurons within the autoradiographically identified transplants.

In terms of the extension and maintenance of pyramidal tract axons, the transplanted neurons behave like the host neurons of the recipient cortical region rather than like those of their site of origin. At P40, following FB injections into the pyramidal decussation on P34, pyramidal tract neurons are labeled within the $\mathbf{O} \rightarrow \mathbf{R}$ transplants, but none can be labeled within $\mathbf{R} \rightarrow \mathrm{O}$ transplants, although in the same $\mathbf{R} \rightarrow O$ cases transplanted neurons are labeled by an injection of DY in the superior colliculus. However, at P13 pyramidal tract neurons can be identified within the $R \rightarrow O$ transplants, as well as in the host occipital cortex, following injections made on P9, a period when the distribution of pyramidal tract neurons in normal rats is widespread (Stanfield and O'Leary, 1985b).

In a second series of host rats, on P34 FB was injected in the pyramidal decussation of the $\mathbf{O} \rightarrow \mathbf{R}$ cases, or in the superior colliculus of the $R \rightarrow O$ cases, and in both groups

\footnotetext{
Received Jan. 15, 1988; revised Sept. 14, 1988; accepted Sept. 20, 1988.

We thank M. A. Lawrence, S. Prausnitz, and R. Granich for histological assistance. This work was supported in part by NEI Grant EY-07025, a McKnight Scholars Award, and an Alfred P. Sloan Fellowship (to D.D.M.O'L.).

Correspondence should be addressed to Dr. Dennis D. M. O'Leary, Department of Neurosurgery, Box 8057, Washington University School of Medicine, 660 South Euclid Avenue, St. Louis, MO 63110.

Copyright (C) 1989 Society for Neuroscience $0270-6474 / 89 / 072230-17 \$ 02.00 / 0$
}

DY was injected into the region of contralateral cortex homotopic for the new location of the transplant. On P40, in both the $O \rightarrow R$ and $R \rightarrow O$ transplants, many neurons singly labeled with FB or DY are found, but no double dye-labeled cells are seen. Thus, as in normal rat cortex, in the transplants the population of callosally projecting neurons is distinct from the population of corticotectal and pyramidal tract neurons, suggesting that the transplanted neurons retain their original neuronal class identity and extend axons to the same set of targets they would have if left in situ.

These findings suggest that the position of a neuron within the tangential plane of the neocortex is a critical factor in determining which of its initially extended axon collaterals will be eliminated and which will be retained as a mature projection. Further, they support the concept that the same classes of projection neurons are present throughout the tangential plane of the early neocortex. From this we infer that the limited distributions of projection neurons characteristic of the adult cortex may not be due to regional differences in the capacity of the neocortical neuroepithelium to generate the complete set of neuronal classes found in the cortex, but rather are the result of the selective elimination of axon collaterals mediated by regionally specific influences.

Studies of the development of cortical projection systems conducted in a number of mammalian orders have established that the limited distributions of projection neuron populations within the tangential plane of the adult neocortex emerge during development from widespread, continuous patterns (see Discussion). In several instances it has been shown that this change in distribution comes about largely, if not entirely, through the selective elimination of axon collaterals, which results in a reduction in the number of major collaterals a cortical projection neuron permanently maintains (Innocenti, 1981; O'Leary et al., 1981; Ivy and Killackey, 1982; Stanfield et al., 1982; Crandall et al., 1985; O'Leary and Stanfield, 1986a). For example, an occipital cortical layer $\mathrm{V}$ cell may initially extend a pyramidal tract collateral as well as a corticopontine and corticotectal collateral; subsequently, the pyramidal tract collateral is lost, while the corticopontine and/or corticotectal projection is retained (O'Leary and Stanfield, 1985). It has also become clear that from the earliest times they can be retrogradely labeled, the projection neuron populations characteristic of the neocortex are segregated into certain distinct classes. For example, neurons that extend a long corticofugal axon to the superior colliculus, 
pons, or spinal cord never have a callosal collateral as well (O'Leary and Stanfield, 1985; Koester and O'Leary, 1989). Thus, the final set of targets of a cortical projection neuron seems to be the result of, first, the projection neuron class to which it belongs, which dictates the initial set of targets, and second, a process of collateral elimination that selectively reduces the targets contacted.

These features of cortical development are consistent with the idea that the full complement of cortical projection neuron classes are produced throughout the tangential extent of the neocortical neuroepithelium but that regional differences extrinsic to the cortical neurons and acting on the process of selective collateral elimination influence the determination of which of the projections initially developed by members of a projection neuron class at a given cortical locale will be maintained.

A test of this conception of cortical development would be to examine the projections extended and maintained by heterotopically transplanted pieces of developing cortex and determine if these resemble those of the region of origin of the transplant or are more appropriate for its new locale. It has become apparent that transplantation - a technique of historically great significance in experimental embryology - can be applied to the mammalian neocortex in such a way. Pieces of fetal cortex transplanted into the brains of neonates continue to generate neurons, appear to mature in a near normal fashion (Lund et al., 1978; Jaeger and Lund, 1980, 1981; Floeter and Jones, 1985; Chang et al., 1986), and are capable of forming connections with the host brain (Chang et al., 1984; Castro et al., 1987).

We have previously shown that pieces of rat fetal occipital cortex transplanted to a rostral cortical position will extend and, for at least the first 4 postnatal weeks, maintain a pyramidal tract projection (Stanfield and O'Leary, 1985a)-a projection initially developed by all regions of the neocortex, and retained by mature rostral cortex but not by mature occipital cortex (Stanfield et al., 1982; Stanfield and O'Leary, 1985b). We now extend this finding by providing evidence that such occipital to rostral cortical transplants permanently maintain a pyramidal tract projection and that rostral to occipital transplants initially develop but subsequently eliminate a pyramidal tract projection. We also demonstrate that the transplanted neurons display other connectional features that are characteristic of both their new cortical locale in the host brain and the specific classes of cortical projection neurons under consideration. Preliminary communications of portions of this work were presented at the annual meetings of the American Association of Anatomists (O'Leary and Stanfield, 1986a) and the Society for Neuroscience (O'Leary and Stanfield, 1986b, c).

\section{Materials and Methods}

Animals. The fetuses and offspring of artificially inseminated female Sprague-Dawley rats purchased from Zivic-Miller were used for all of the experiments. The day of insemination is designated as embryonic day (E) 0 . The pups were usually born on E22. The first $24 \mathrm{hr}$ after birth is designated postnatal day (P)0.

Transplantations. Pregnant females were given a single intraperitoneal injection of $\mathrm{Me}-{ }^{3} \mathrm{H}$-thymidine $\left({ }^{3} \mathrm{H}-\mathrm{TdR}, 10 \mu \mathrm{Ci} / \mathrm{gm}\right.$ body weight; specific activity, $6.7 \mathrm{Ci} / \mathrm{mmol}$; New England Nuclear) on E15 to mark the transplant for later identification in the host brain.' On E17 the pregnant rat was anesthetized; the fetuses were removed and decapitated, and their brains were dissected out and placed in Ham F10 (Gibco) medium.

'El 5 was chosen because most cortical neurons are generated between E14 and E19 (Lund and Mustari, 1977), and layer $V$ neurons in particular are generated primarily on E15 and E16 (Bruckner et al., 1976).

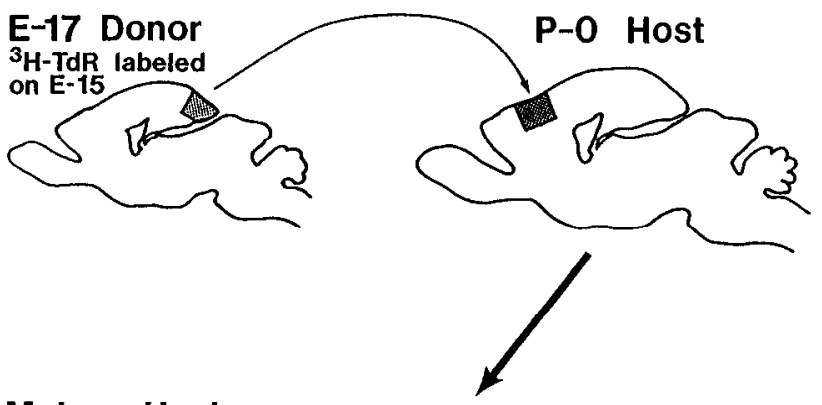

Mature Host

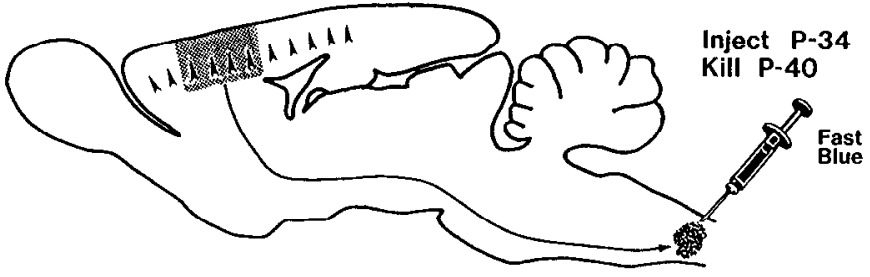

Figure 1. Schematic illustration of the paradigm used to determine that a piece of posterior occipital cortex transplanted to a rostral cortical region maintains a pyramidal tract projection well after the time the transient pyramidal tract projection from the occipital cortex is lost. The results are documented in Figures 2-5.

A small piece of cortex, containing the full radial thickness of the cortex from the ventricular surface to the pial surface, was cut out of either the rostral region or the posterior occipital region at the caudal pole of the cerebral hemisphere from both the left and right sides of each donor brain (Fig. 1), the pia removed, and the piece of cortex trimmed further. We were careful to use only posterior occipital cortex for transplantations, since the very rostral part of the occipital region immediately adjacent to the parietal cortex does contain a small number of pyramidal tract neurons in the adult rat (Miller, 1987; Terashima and O'Leary, 1988). The host P0 pup was anesthetized by hypothermia and its scalp opened. Incisions were made into the skull overlying a discrete region of the right cortical hemisphere. The skull flap was reflected, and a small hole was made by aspiration in either rostral cortex (i.e., frontal or rostral parietal cortex, for occipital to rostral cases) or in posterior occipital cortex (for rostral to occipital cases). We attempted to remove all or most of the depth of the cortical gray while leaving the underlying white matter intact. The piece of donor cortex was placed in the hole and the skull flap was put back in place. Care was taken to place the donor piece in the correct superficial/deep orientation. The scalp wound was sutured and sealed with a cyanoacrylate ester glue. Each pup was uniquely marked to allow for later identification, and after recovering from anesthesia returned to its mother. The donor brains were fixed by immersion in Carnoy's solution.

In 9 newborn rats, a small aspiration lesion was made in rostral cortex in an identical fashion to that described above, but a piece of donor cortex was not placed into the cavity. The surgery was then completed as if a transplantation were performed. On P35, these animals were injected with Fast blue (FB) in the pyramidal decussation and perfused $6 \mathrm{~d}$ later, as described below for the occipital to rostral transplant cases.

Tracer injections. The retrogradely transported fluorescent dyes FB (Bentivoglio et al., 1980) and Diamidino yellow dihydrochloride (DY; Keizer et al., 1983) were used to examine the distribution of populations of cortical projection neurons. Both dyes were used as $2 \%$ suspensions in distilled water and were injected with separate $1 \mu$ l Hamilton syringes to which a 27 gauge blunt-tipped needle was attached. The host rats were injected at 1 of 2 different ages, P34 and P9. In one group of P34 host rats consisting of animals that had received either a rostral to occipital or an occipital to rostral transplant, FB was used to label pyramidal tract neurons. For this, the animals were anesthetized with chloral hydrate $(3.5 \mathrm{mg} / \mathrm{gm}$ body weight). An incision was made at the back of the skull, the muscles and the occipito-atlantal ligament were reflected, and the underlying dura was cut and moved aside to expose the caudal brain stem. The injection needle was lowered into the pyramidal decussation at the junction of the medulla and cervical spinal cord and $0.5-1.0 \mu 12 \% \mathrm{FB}$ was injected. The right superior colliculus 


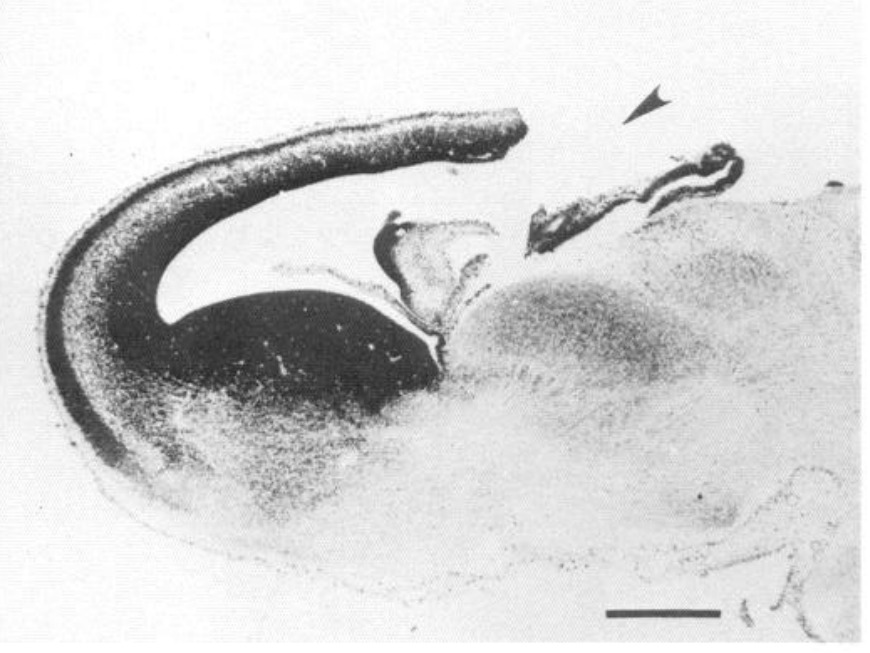

Figure 2. Nissl-stained sagittal section taken from the donor brain used for the case illustrated in Figure 3. The position at the posterior occipital pole from which the transplant was removed is indicated by the arrowhead. Scale bar, $0.5 \mathrm{~mm}$.

(ipsilateral to the transplant) of the rostral to occipital host rats in this group was also injected at this time with DY to label corticotectal neurons. To avoid an incidental labeling of the cortex that may result from the injection needle passing through it, an angled approach was used. A small hole was made in the skull overlying the cerebellum through which the injection needle was passed at a $50^{\circ}$ angle. The needle tract continued through the cerebellum and inferior colliculus, and 0.2 $\mu$ l $2 \%$ DY was injected into the superior colliculus. The animals were sutured and returned to their cages.

In another group of P34 host rats, FB was injected into the pyramidal decussation of the occipital to rostral cases and into the superior colliculus of the rostral to occipital cases as described above. In addition, to label callosally projecting neurons in both sets of cases, DY was injected on P34 into the region of the contralateral cortex homotopic to the site of the transplant. For this, 5-6 small holes spaced to cover much of either rostral or occipital cortex were made in the skull, and $0.1-0.3 \mu \mathrm{l} 2 \%$ DY was injected in each site.

All rats injected on P34 were killed $6 \mathrm{~d}$ later. The rats were deeply anesthetized with chloral hydrate and perfused transcardially with $0.9 \%$ saline followed by a solution of $10 \%$ formalin in $0.1 \mathrm{~m}$ phosphate buffer (pH 7.4). The brains were removed from their skulls, placed into the fixative to which $10 \%$ sucrose had been added, and stored at $4^{\circ} \mathrm{C}$.

A third group of host rats, all of which were rostral to occipital cases, were injected with $0.3 \mu \mathrm{l} 2 \% \mathrm{FB}$ into the pyramidal decussation as above, but on P9, and were perfused on P13.

Histology and analysis. The host brains were frozen-sectioned at 40 $\mu \mathrm{m}$ on a sliding microtome, and two 1 -in-5 series of sections were mounted out of $0.1 \mathrm{M}$ phosphate buffer onto gelatin-coated slides and air-dried. The sections were examined and photographed on a microscope equipped with epifluorescence attachments, a $100 \mathrm{~W}$ mercury light source, and the appropriate UV filter cube (excitation filter, 340$380 \mathrm{~nm}$; barrier filter, $430 \mathrm{~nm}$ ). The remaining sections were stored in $2 \%$ buffered formalin.

For those cases in which the injections of the retrograde dyes were correctly targeted, a third 1-in-5 series of sections was subsequently mounted out of gelatin-alcohol onto gelatin-coated slides and air-dried. This series was defatted in chloroform-alcohol and dipped in Kodak NTB-2 emulsion for autoradiography. One or both of the remaining 2 series of sections were occasionally processed as well. After a 4 week exposure at $4^{\circ} \mathrm{C}$, the slides were developed in $\mathrm{D} 19$ at $16^{\circ} \mathrm{C}$, fixed in Ektaflo, and coverslipped using a nonfluorescent buffered glycerol mountant. The cases were reexamined and the transplants verified with bright- and dark-field optics by the accumulation of silver grains in the overlying emulsion, indicative of ${ }^{3} \mathrm{H}$-TdR-labeled cells in the donor tissue. Successful cases were photographed under both fluorescence and dark-field illumination. Selected sections were then counterstained with thionin and rephotographed under bright- and dark-field illumination.
The donor brains were dehydrated through a graded series of alcohols, cleared in xylene, and embedded in paraffin. Those from successful cases were subsequently sectioned at $15 \mu \mathrm{m}$ on a rotary microtome. A 1-in5 series of sections was mounted with albumin onto clean glass slides, deparaffinized, and processed for autoradiography in the manner described by Cowan et al. (1972). Following a 4 week exposure, the slides were developed, and the sections counterstained with thionin and coverslipped with DPX. The sections were examined and photographed with bright- and dark-field optics.

\section{Results}

Evidence that the retention or elimination of pyramidal tract axons by heterotopic cortical transplants is dependent on the new cortical locale

In our previous study (Stanfield and O'Leary, 1985a), we found that fetal occipital cortical neurons transplanted to a rostral cortical locale in a newborn rat pup could be shown to have established a pyramidal tract projection when the spinomedullary junction was injected with the retrograde tracer FB on P25 and examined at P30. This evidence suggests that when transplanted during development cortical neurons form and maintain projections appropriate to their new, rather than original, locale. However, since during normal development the occipital cortex does transiently extend axons through the pyramidal tract, all of which are eliminated by the middle of the third postnatal week (Stanfield et al., 1982; Stanfield and O'Leary, 1985b), an alternate possibility is that the normal progression of cortical development is delayed in the transplanted tissue (see, for example, Herman et al., 1988). We have approached this issue in 2 ways. The first was simply to repeat our previous paradigm of transplanting occipital cortex to a rostral cortical locale but now allowing the animals to survive for a longer period of time prior to assaying for the presence of pyramidal tract neurons within the transplant. The second and perhaps more critical approach was to transplant fetal rostral cortex into an occipital locale and to determine if such transplants could establish a pyramidal tract projection and, if so, whether this projection is maintained (as it is during the normal development of the rostral cortex) or lost (as it is during the normal development of the occipital cortex).

\section{Occipital to rostral transplants}

The experimental paradigm for the occipital to rostral transplants is outlined schematically in Figure 1. Briefly, a small piece of posterior occipital cortex was removed from the caudal pole of the cerebral hemisphere of a ${ }^{3} \mathrm{H}$-TdR-labeled E1 7 donor brain and transplanted to a rostral cortical region (i.e., frontal or rostral parietal cortex) of a newborn rat pup. On P34, $0.5 \mu \mathrm{l}$ of a $2 \%$ suspension of FB was injected into the pyramidal decussation, and the animal was perfused $6 \mathrm{~d}$ later. Of the 23 animals of this paradigm that survived to maturity, 19 had an identifiable transplant. In 13 of these, the FB injection was confined to the spinomedullary junction and judged successful by the degree of labeling seen in host rostral cortex. In all cases no labeled neurons were present in the caudal third of the neocortex, including the visual cortex. FB-labeled neurons were found in 8 of the transplants.

The primary finding from this series of experiments is that occipital cortical neurons transplanted to a rostral cortical locale will not only extend pyramidal tract axons, but will maintain them at least through the sixth postnatal week. Figure 2 is a photomicrograph of the donor brain for one of these cases and shows the region of occipital cortex removed for transplantation. 

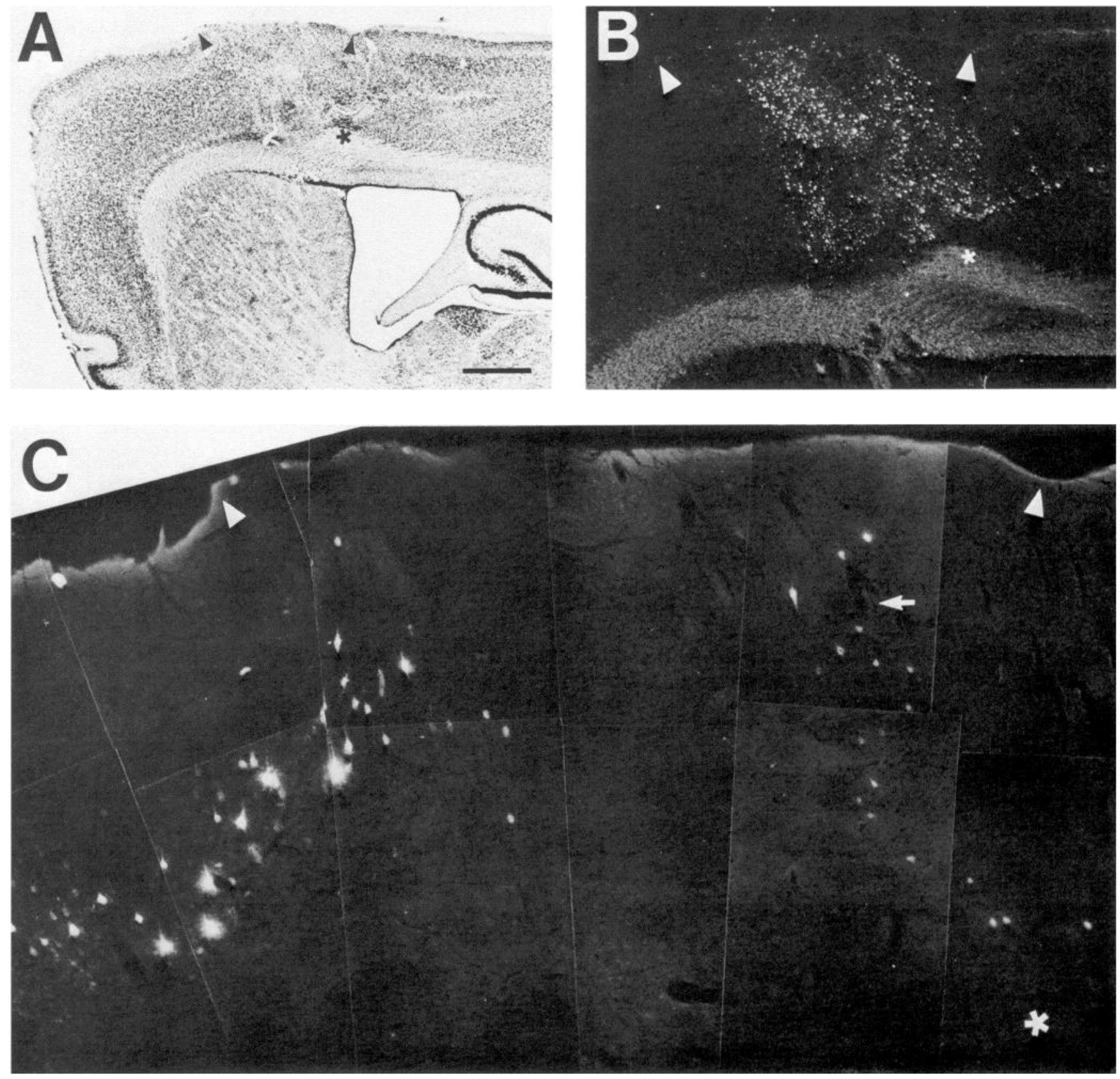

Figure 3. A-C are bright-field, dark-field, and fluorescence photomicrographs of the same sagittal section taken from a host brain in which a piece of posterior occipital cortex was transplanted to rostral cortex. The arrowheads mark the same features on the surface of the brain and the asterisk indicates the same point in the white matter in $A-C$. The location and Nissl-stained appearance of the ${ }^{3} \mathrm{H}-\mathrm{TdR}-\mathrm{labeled}$ transplant is shown in $A$, and its boundaries can be delineated on the basis of the accumulations of silver grains seen in the autoradiogram shown in $B$. In $C$, neurons retrogradely labeled with FB injected into the pyramidal decussation are seen both within the host cortex and the transplant. All of the FB-labeled neurons on the right half of $C$ are in the transplant. The region of the transplant indicated by the arrow in $C$ is shown at a higher power in Figure 4. Rostral is to the left, dorsal to the top. Scale bar: $A, 1 \mathrm{~mm} ; B, 0.75 \mathrm{~mm} ; C, 0.2 \mathrm{~mm}$.

Figure $3 A$ shows the position of this transplant within the host brain. In this particular case, the transplant is located in frontal cortex. The boundaries of the transplant can be accurately delineated in autoradiograms of sections of the host brain from the distribution of silver grain clusters indicative of ${ }^{3} \mathrm{H}-\mathrm{TdR}$ labeled cells from the donor brain (Fig. $3 B$ ). The transplant extends from the pial surface down to the deep cortical white matter. A large number of pyramidal tract neurons retrogradely labeled with FB injected into the spinomedullary junction (Fig.
4) can be seen not only in layer V of the host cortex, but within the transplant as well (Fig. 3C). As in host cortex, most of the FB-labeled neurons in the transplant have a pyramidal shape (Fig. $3 C$ ). In this case, the FB-labeled neurons are not scattered throughout the transplant, but rather form a continuous band, although this band of labeled cells does not parallel the cortical surface as in host cortex (likely because of a misalignment of the transplant with the host cortex). Thus, the normal lamination of the cortex appears to have been maintained, at least to 


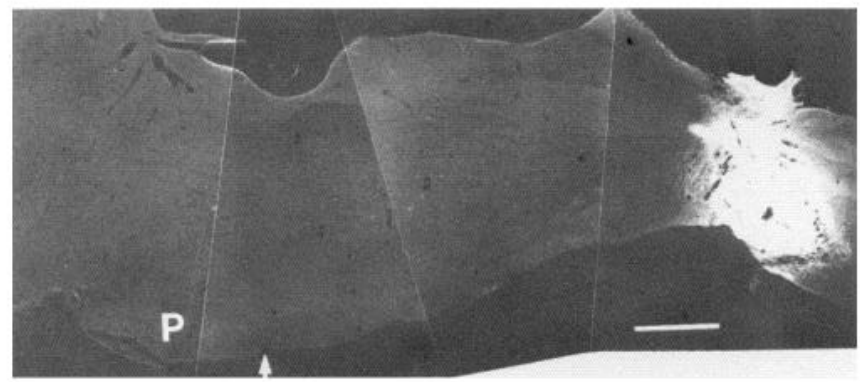

Figure 4. Fluorescence photomontage of a sagittal section through the center of the FB injection site from the case illustrated in Figure 3. The injected FB is confined to the region of the pyramidal decussation at the spinomedullary junction. The injected tracer did not spread rostral to this point, $5 \mathrm{~mm}$ posterior to the caudal limit (arrow) of the basilar pons $(P)$. Rostral is to the left, dorsal to the top. Scale bar, $1 \mathrm{~mm}$.

some extent, within the transplant. Many of the FB-labeled neurons within the transplant of this, as well as the other successful cases, are also labeled with ${ }^{3} \mathrm{H}-\mathrm{TdR}$ (Fig. 5), indicating that they did, in fact, originate from the donor tissue. We have not attempted to determine the percentage of FB-labeled neurons that were also labeled with ${ }^{3} \mathrm{H}-\mathrm{TdR}$, but we would expect that it would be a low figure, since only a proportion of pyramidal tract neurons would be labeled by the pulse of ${ }^{3} \mathrm{H}-\mathrm{TdR}$ on E15 (Bruckner et al., 1976; O'Leary and Stanfield, unpublished observations), and of these, only those ${ }^{3} \mathrm{H}$-TdR-labeled nuclei located within the upper 2-3 $\mu \mathrm{m}$ of the $40-\mu \mathrm{m}$-thick sections would have silver grains overlying them (Rogers, 1973). Nor have we quantified the proportion of ${ }^{3} \mathrm{H}$-TdR-labeled cells that are also dye-labeled since pyramidal tract neurons and several other classes of cortical neurons are generated concurrently.

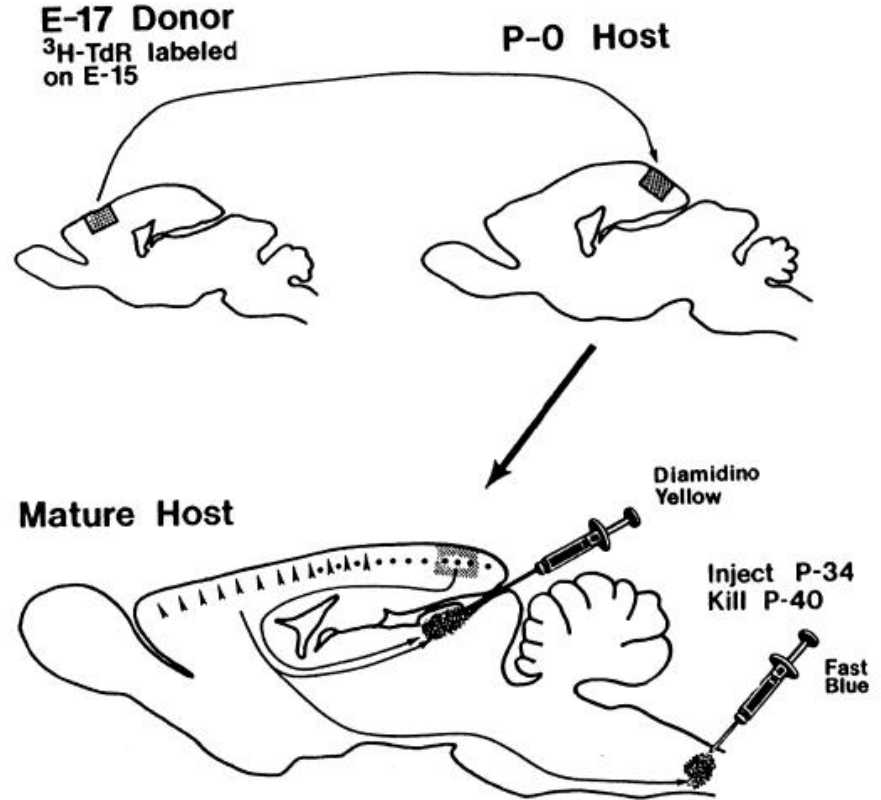

Figure 6. Schematic diagram of the experimental paradigm used to determine whether a piece of rostral cortex transplanted to the occipital region will maintain a pyramidal tract projection or a corticotectal projection. The donor brain and the results from a representative case are illustrated in Figures 7-9A, 10.

\section{Rostral to occipital transplants}

The experiments involving rostral to occipital transplants can be subdivided into 2 groups: cases tested for the development and maintenance of pyramidal tract and corticotectal projections and cases tested after a relatively brief survival period for
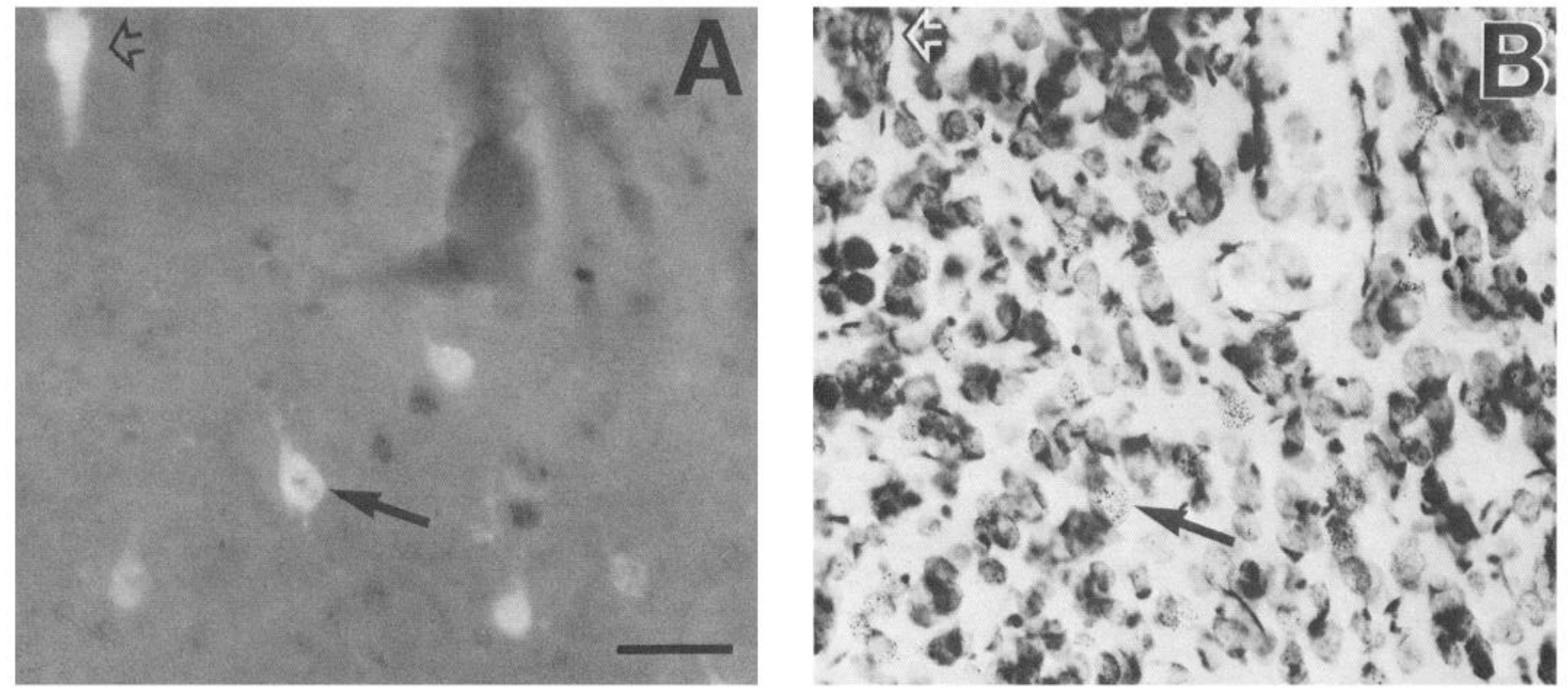

Figure 5. Higher-magnification view of the region of the transplant indicated by the arrow in Figure $3 C$. $A$ is a fluorescence photomicrograph of several FB-labeled pyramidal tract neurons, 2 of which are marked by arrows. In $B$, the same 2 neurons are marked by arrows in the bright-field photomicrograph of the Nissl-stained autoradiogram of the same section. An accumulation of silver grains overlies the nucleus of the neuron marked by the solid arrow indicating that it is also labeled with ${ }^{3} \mathrm{H}-\mathrm{TdR}$. Rostral is to the left, dorsal to the top. Scale bar, $50 \mu \mathrm{m}$. 


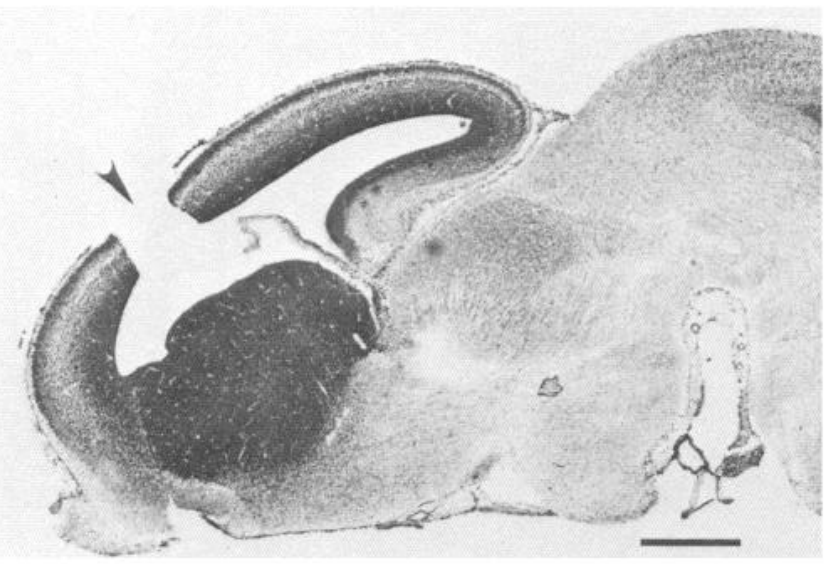

Figure 7. Bright-field photomicrograph of a Nissl-stained sagittal section through the donor brain for the rostral to occipital case shown in Figures 8, 9A, 10. The arrowhead indicates the region of rostral cortex from which the transplant was taken. Rostral is to the left, dorsal to the top. Scale bar, $0.5 \mathrm{~mm}$.

the initial extension of pyramidal tract axons. When taken together, the findings from these 2 groups of experiments indicate that rostral cortical neurons transplanted to occipital cortex initially extend pyramidal tract axons but subsequently eliminate them.

The experimental paradigm for the first group is outlined in Figure 6. A piece of rostral cortex was removed from the brain of an E17 rat fetus and transplanted to the posterior occipital cortical region of a newborn. The donor tissue had been exposed to ${ }^{3} \mathrm{H}-\mathrm{TdR}$ on the 15 th day of gestation by means of a maternal intraperitoneal injection. On P34, the host animal was injected with 2 different retrogradely transported fluorescent dyes: FB into the pyramidal decussation and DY into the superior colliculus, a known target of layer $\mathrm{V}$ neurons in both rostral (Wise and Jones, 1977) and occipital (Olavarria and van Sluyters, 1982) cortex. The rats were perfused on P40. A transplant was identified in 41 of the 63 host animals that survived until P40. In 26 of the cases with a transplant, the FB injection was successful and resulted in a band of well-labeled pyramidal tract neurons within the rostral two-thirds of the cortex. In none of these cases did we observe any FB-labeled neurons in the transplant. On the other hand, in 9 of these cases in which the DY injection into the superior colliculus was successful, DY-labeled neurons were present in both the host occipital cortex and in the transplant. An example of one of these cases is illustrated in Figures 7-9 $A, 10$. For this particular case, the region of rostral cortex removed from the donor brain for transplantation is shown in Figure 7. The location of the transplant near the occipital pole of the host brain, and the corresponding autoradiogram used to delineate the borders of the transplant, are shown in Figures $8, A$ and $B$, respectively. The transplant extends the full radial thickness of the cortex, from the pial surface to the underlying cortical white matter.

The distribution within the host cortex of neurons labeled by the injections of the 2 different dyes is similar to that seen in normal rats. The FB injected into the pyramidal decussation labeled a dense band of layer $\mathrm{V}$ neurons confined to about the rostral two-thirds of the host cortex, whereas the DY injected into the superior colliculus labeled a prominent band of layer $\mathrm{V}$ neurons within both host occipital cortex, as well as in more
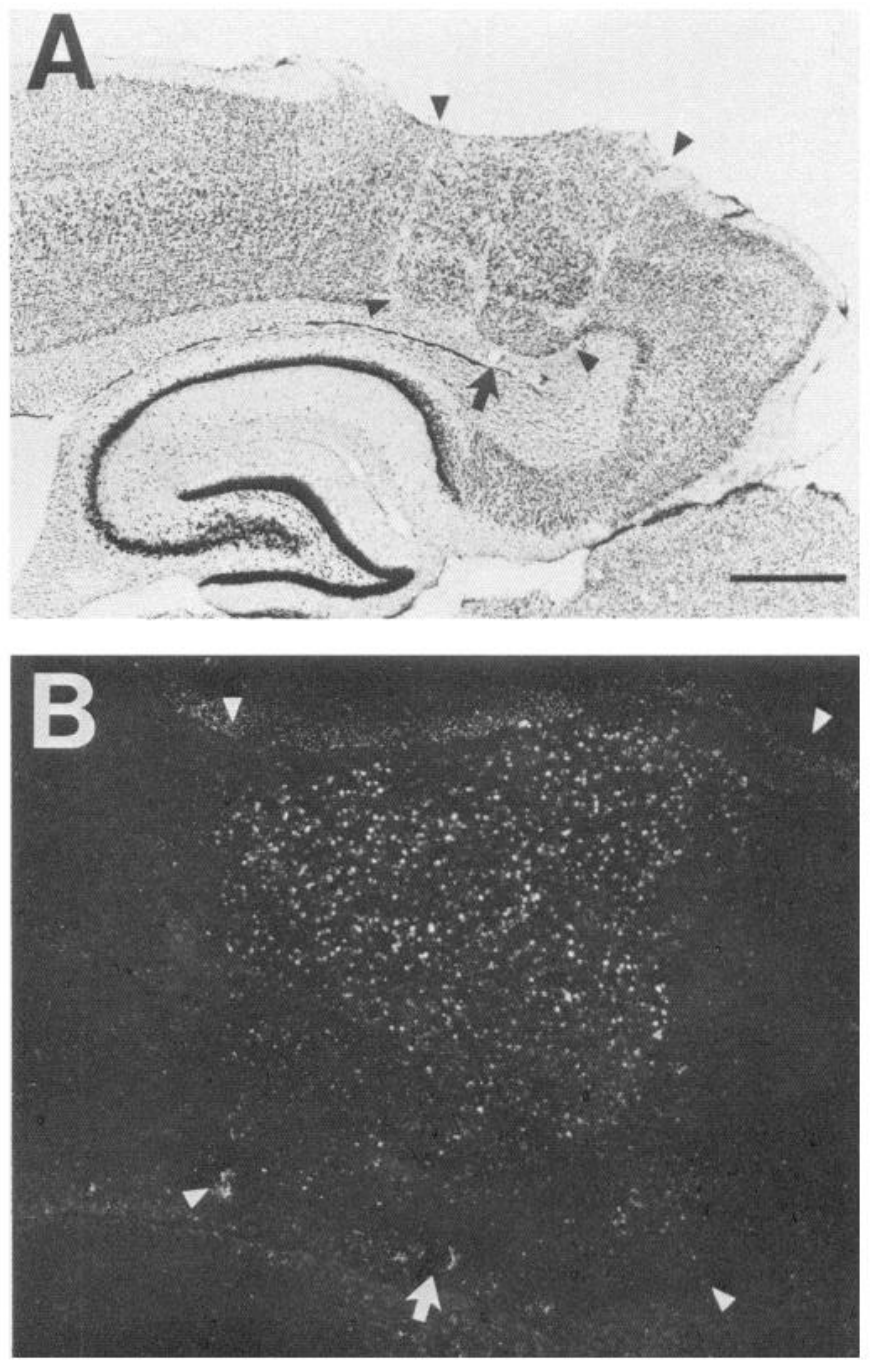

Figure 8. $A$ and $B$, Bright- and dark-field photomicrographs, respectively, of a Nissl-stained autoradiogram of a sagittal section through the posterior part of the cortex of the host brain of a rostral to occipital transplant case. The ${ }^{3} \mathrm{H}-\mathrm{TdR}$-labeled transplant can be identified in $B$ on the basis of the accumulations of silver grains. The arrowheads mark the borders of the transplant, and the arrow points to the same blood vessel in both $A$ and $B$. Rostral is to the left, dorsal to the top. Scale bar: $A, 750 \mu \mathrm{m} ; B, 300 \mu \mathrm{m}$.

rostral cortical fields. Figure $9 A$ illustrates the distribution of the dye-labeled neurons within the transplant and adjacent regions of host cortex. A band of FB-labeled pyramidal tract neurons is seen confined to the host parietal cortex and the extreme rostral part of the occipital cortex. No FB-labeled neurons are present within the transplant, nor in the host occipital cortex caudal to the transplant. However, the band of DY-labeled corticotectal neurons spans from the host parietal cortex, into the transplant, and back into host occipital cortex, where it continues uninterrupted to the caudal pole of the cortex. A substantial number of DY-labeled neurons are present in the transplant, and although the laminar arrangement of these cells is not as compact as in the host occipital cortex, they are largely restricted to a layer rather than distributed throughout the transplant. As in the occipital to rostral transplants, in all of the successful cases of this paradigm, many of the dye-labeled cells in the 

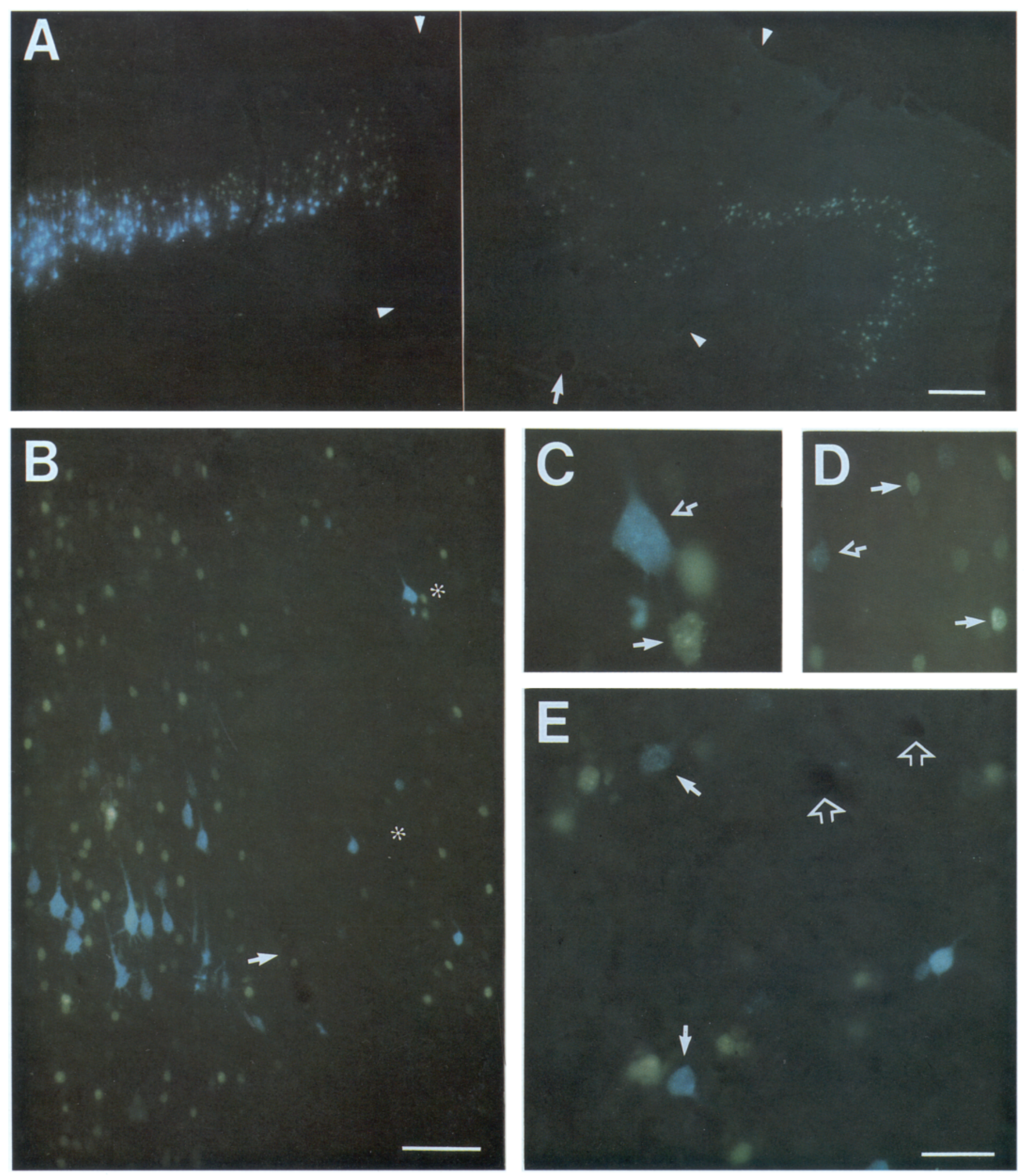

Figure 9. A, Fluorescence photomontage of the same section illustrated in Figure 8. The arrowheads mark the borders of the rostral to occipital transplant, and the arrow points to the same blood vessel indicated by arrows in Figure 8. DY-labeled corticotectal neurons (yellow-green dots) form a nearly continuous band extending from the host parietal cortex rostral to the transplant, through the transplant, and into the host occipital cortex caudal to the transplant. The band of FB-labeled neurons (bluish color) is strictly limited to the region of host cortex rostral to the transplant. Rostral is to the left, dorsal to the top. Scale bar, $250 \mu \mathrm{m}$. B, Fluorescence photomicrograph of the section presented in Figure 17A showing at higher power the region of the border of host cortex and the transplant; the right half is transplant tissue, the left half is host cortex. The arrow marks the same blood vessel as in Figure 17A. Neurons singly labeled with either FB injected into the pyramidal tract (bluish color) or DY injected 

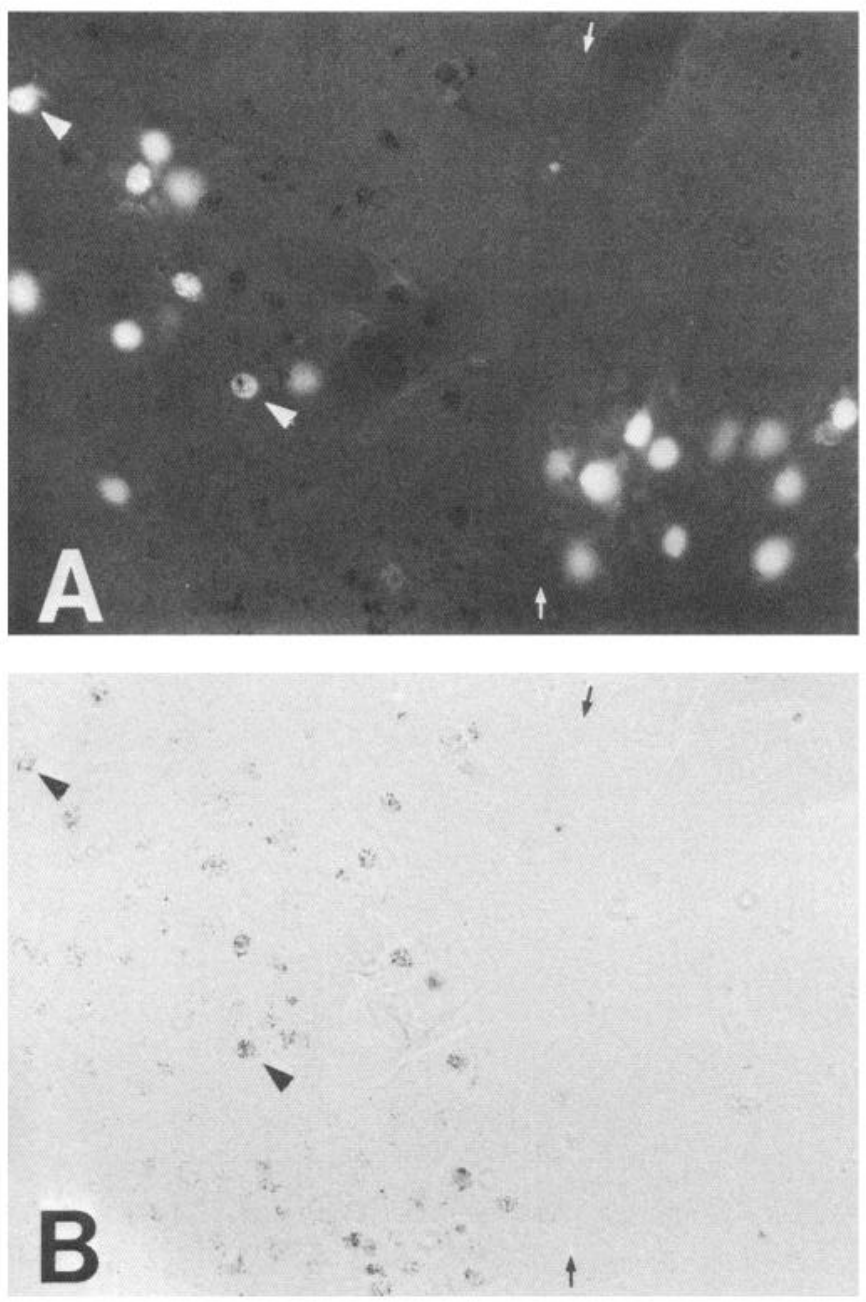

Figure 10. Fluorescence $(A)$ and bright-field $(B)$ photomicrographs of an autoradiogram of a sagittal section from the same case illustrated in Figure $9 A$. The arrows mark the caudal border between the rostral to occipital transplant (to the left-note the large number of ${ }^{3} \mathrm{H}-\mathrm{TdR}$ labeled cells) and host occipital cortex. Most of the DY-labeled corticotectal neurons within the transplant are also labeled with ${ }^{3} \mathrm{H}-\mathrm{TdR}$ as indicated by the accumulations of silver grains over the DY-labeled nuclei. Two of these double-labeled neurons are marked by arrowheads in $A$ and $B$. Rostral is to the left, dorsal to the top.

transplant are also labeled with ${ }^{3} \mathrm{H}-\mathrm{TdR}$ (Fig. 10), verifying that they are of donor origin.

This set of experiments indicates that a piece of fetal rostral cortex transplanted to posterior occipital cortex can establish and maintain a projection to the superior colliculus but does not maintain a pyramidal tract projection. Since normally the occipital cortex transiently projects axons through the pyramidal

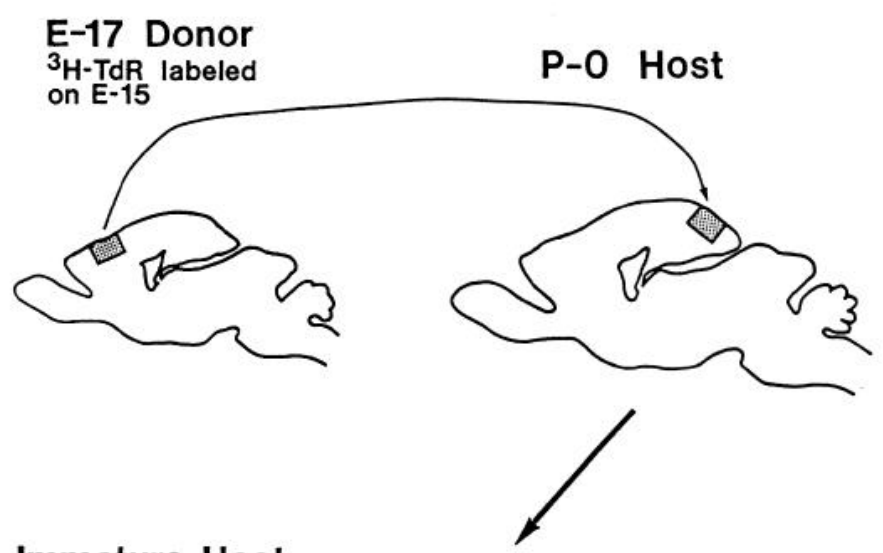

Immature Host

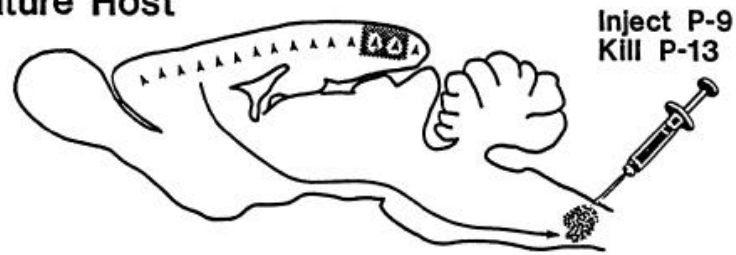

Figure 11. Schematic diagram of the experimental paradigm used to determine that a piece of rostral cortex transplanted to the occipital region initially develops a pyramidal tract projection. The donor brain and results from a representative case are illustrated in Figures 12-15.

tract, in order to determine whether rostral to occipital transplants initially form a pyramidal tract projection that is not maintained, we carried out the group of experiments schematically outlined in Figure 11 . As before, a piece of ${ }^{3} \mathrm{H}-\mathrm{TdR}$ labeled rostral cortex was removed from an E17 donor brain and transplanted to the occipital region of a newborn rat. But in these cases, FB was injected into the pyramidal decussation of the host rats on P9, and the animals were perfused on P13. Thus, the host rats were injected and killed prior to the elimination of the transient occipital pyramidal tract projection in normal rats (Stanfield and O'Leary, 1985b).

Of the 34 host rats that were injected, 20 had an identifiable transplant. Of these 20 cases, 8 had injections confined to the spinomedullary junction and adequate $\mathrm{FB}$ labeling of pyramidal tract neurons in the host cortex, 5 of which had FB-labeled neurons in the transplant. The results from one such case are illustrated in Figures 12-15. The site of origin of this transplant is shown in Figure 12. As determined from Nissl-stained autoradiograms, the transplant is located at the posterior pole of occipital cortex of the host brain (Fig. 13, $A, B$ ). As is normal for rats of this age (Stanfield et al., 1982; Stanfield and O'Leary, $1985 \mathrm{~b}$ ), the pyramidal tract neurons retrogradely labeled from the pyramidal decussation by the FB injection confined to the spinomedullary junction (Fig. 14) are found widely distributed

into the opposite cortex (yellow-green color) are present in both the host cortex and the transplant. Two regions of the transplant marked by asterisks are shown at higher magnification in $C$ and $D$. Rostral is to the left, dorsal to the top. Scale bar, $200 \mu$ m. $C$, Fluorescence photomicrograph of the more dorsal of the 2 regions of the transplant marked by asterisks in $B$. The open arrow points to a pyramidal tract neuron singly labeled with FB, the solid arrow to a DY-labeled callosal neuron. $D$, Fluorescence photomicrograph of the more ventral of the 2 regions of the transplant marked by asterisks in $B$. The open arrow points to a pyramidal tract neuron singly labeled with $\mathrm{FB}$, the solid arrows to DY-labeled callosal neurons. $E$, Appearance of FB-labeled corticotectal neurons (bluish color; some indicated by solid arrows) and DY-labeled callosal neurons (yellow-green color) shown in this fluorescence photomicrograph of the region of the rostral to occipital transplant marked by the asterisk in Figure 19. None of the neurons are labeled with both dyes. The 2 FB-labeled corticotectal neurons marked by solid arrows are also labeled with ${ }^{3} \mathrm{H}-\mathrm{TdR}$ as shown in Figure 20. The open arrows point to the same blood vessels as those in Figure 20. Scale bar, $50 \mu \mathrm{m}$. 


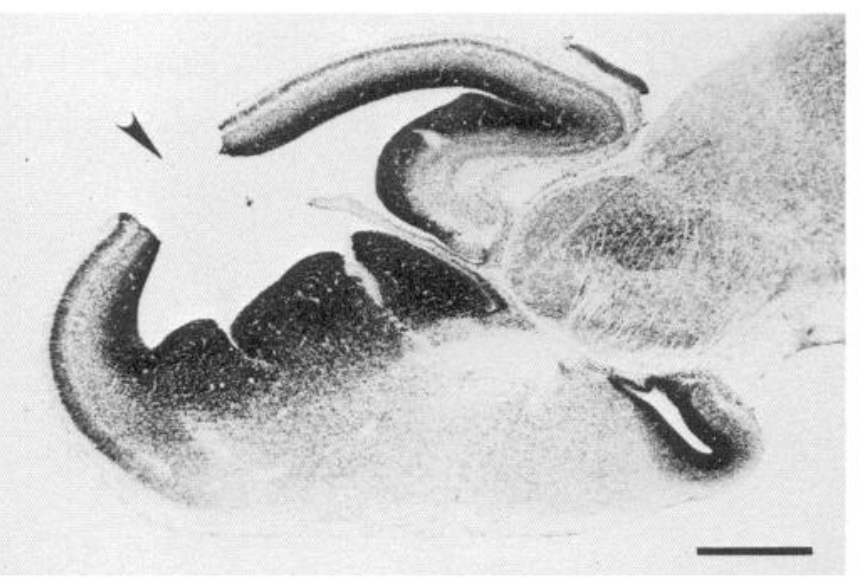

Figure 12. Bright-field photomicrograph of a sagittal section from the donor brain for the rostral to occipital transplant case illustrated in Figures 13-15. The region of rostral cortex from which the transplant was removed is indicated by the arrowhead. Rostral is to the left, dorsal to the top. Scale bar, $0.5 \mathrm{~mm}$.

within the tangential plane of the host cortex. The band of FBlabeled layer $\mathrm{V}$ neurons in more rostral cortical regions continues uninterrupted into and throughout host occipital cortex. In Figure $13 C$, a substantial number of FB-labeled pyramidal tract neurons can be seen to form a well-defined band extending through the transplant. In this case, as in the others, many of the FB-labeled neurons within the transplant are also labeled with ${ }^{3} \mathrm{H}$-TdR (Fig. 15), verifying that they originated from the donor tissue.

When taken together, these 2 groups of experiments indicate that fetal rostral cortical neurons within a transplant placed in occipital cortex behave like host occipital cortical neurons in that they initially extend axons through the pyramidal tract but subsequently eliminate them, while maintaining a projection to the superior colliculus.

\section{Evidence that projection neuron populations within heterotopic cortical transplants are the same as found in normal cortex}

The results of the experiments described in the previous section suggest that heterotopically transplanted pieces of cortex maintain projections appropriate for their new locale. They do not allow us to determine, however, if the neurons that maintain these projections are the same ones that would have transiently extended such collaterals during development. For example, are the cells that maintain pyramidal tract collaterals in the occipital to rostral transplants the same neurons that would have transiently extended such a projection if left in the occipital cortex? At issue is whether following transplantation to a new locale the neurons simply express a projection that is normally within their potential, in that it is appropriate for what we have termed their projection neuron class, or if the procedure is changing the character of the neurons. To approach this issue, we have done 2 additional sets of heterotopic cortical transplant experiments to determine if the transplanted neurons that project to the contralateral cortex are an independent population from those that send an axon through the pyramidal tract or to the superior colliculus, as is the case in the normal cortex of adult rats as well as in neonates before the phase of selective collateral elimination (O'Leary and Stanfield, 1985).
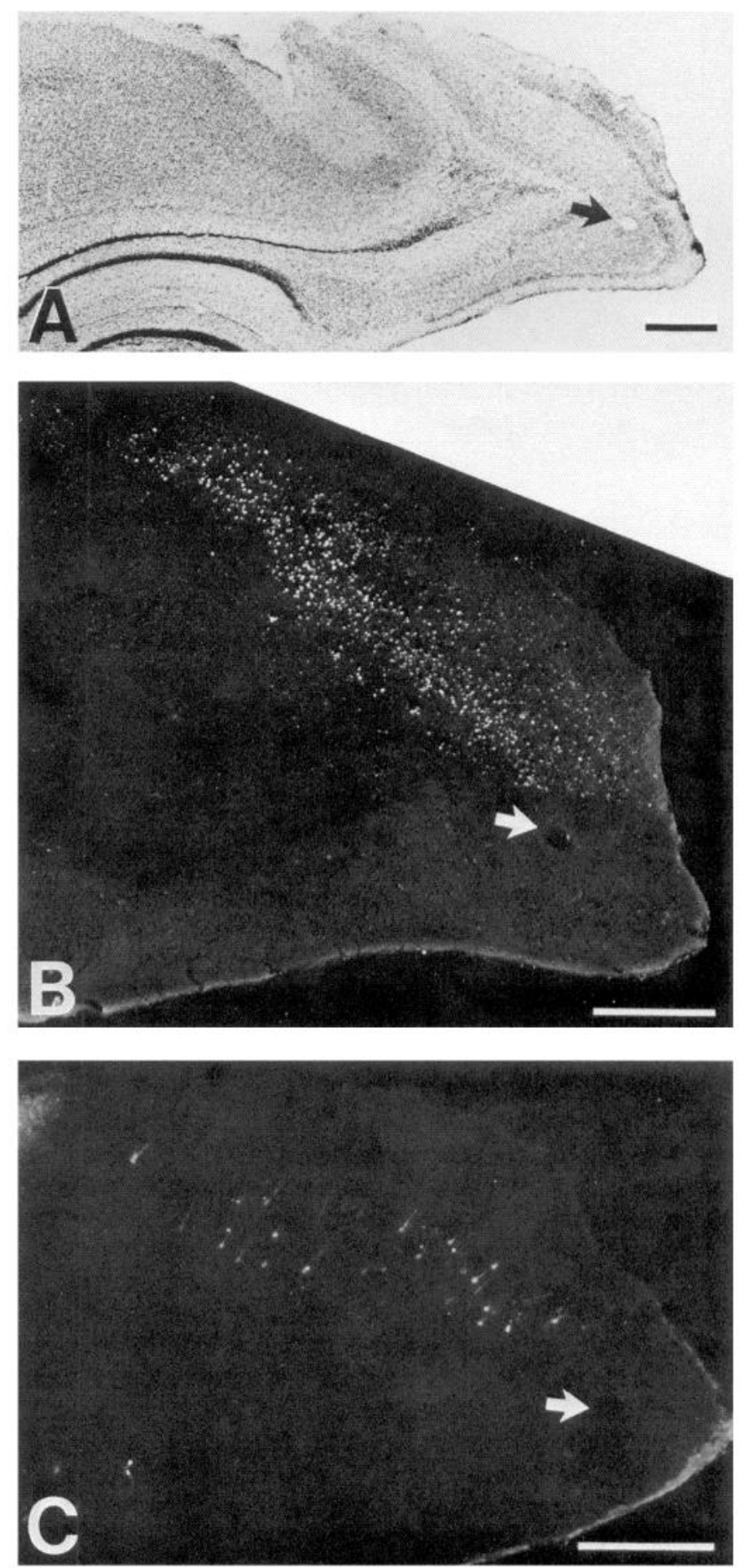

Figure 13. $A$ and $B$, Bright- and dark-field photomicrographs, respectively, of a Nissl-stained autoradiogram of a sagittal section from a P13 rostral to occipital transplant case showing the location of the transplant in posterior occipital cortex. The ${ }^{3} \mathrm{H}-\mathrm{TdR}$-labeled transplant can be identified in $B$ on the basis of the accumulations of silver grains. $C$, Fluorescence photomicrograph of an adjacent section to that in $A$ and $B$ showing a significant number of FB-labeled pyramidal tract neurons within the transplant. The arrow points to the same blood vessel in the 3 panels. Rostral is to the left, dorsal to the top. Scale bars, $0.5 \mathrm{~mm}$.

In the first set of experiments, a piece of posterior occipital cortex was removed from a ${ }^{3} \mathrm{H}-\mathrm{TdR}$-labeled E17 donor brain and transplanted to a rostral cortical locale in a newborn rat. On P34, multiple injections of DY were made into the contra- 


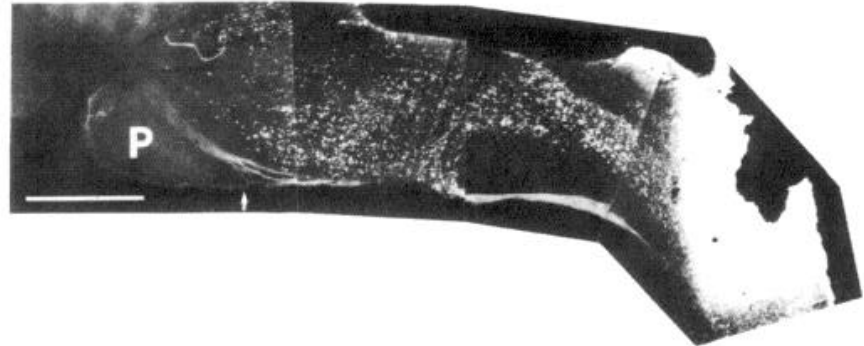

Figure 14. Fluorescence photomontage of a sagittal section through the center of the FB injection site at the spinomedullary junction from the rostral to occipital transplant case illustrated in Figure 13. The basilar pons $(P)$ is $4 \mathrm{~mm}$ rostral to the rostralmost spread of the injected FB. Pyramidal tract axons positioned along the ventral aspect of the medulla and over the basilar pons are retrogradely labeled with FB. The arrow marks the junction between the pons and medulla. Rostral is to the left, dorsal to the top. Scale bar, $1 \mathrm{~mm}$.

lateral rostral cortex, and FB was injected into the pyramidal decussation. The animals were perfused on P40. Within host rostral cortex, both rostral and caudal to the transplants, a band of FB-labeled pyramidal tract neurons was found within layer V. As in normal adult rats, the FB-labeled cells were confined to about the rostral two-thirds of the neocortex. DY-labeled callosally projecting neurons were distributed throughout layers II-VI in the region of cortex homotopic for that injected on the opposite side. Consistent with previous findings (Catsman-Berrevoets et al., 1980; O'Leary and Stanfield, 1985), we did not find any host cortical neurons doubly labeled with the 2 fluorescent tracers.

A distribution of labeled neurons similar to that seen in host cortex was found in many of the transplants. A transplant could be identified in 60 of the 74 host rats. In 47 of the 55 cases with successful DY injections made into the region of contralateral cortex homotopic for the new locale of the transplant, large numbers of DY-labeled neurons were found in the transplant.

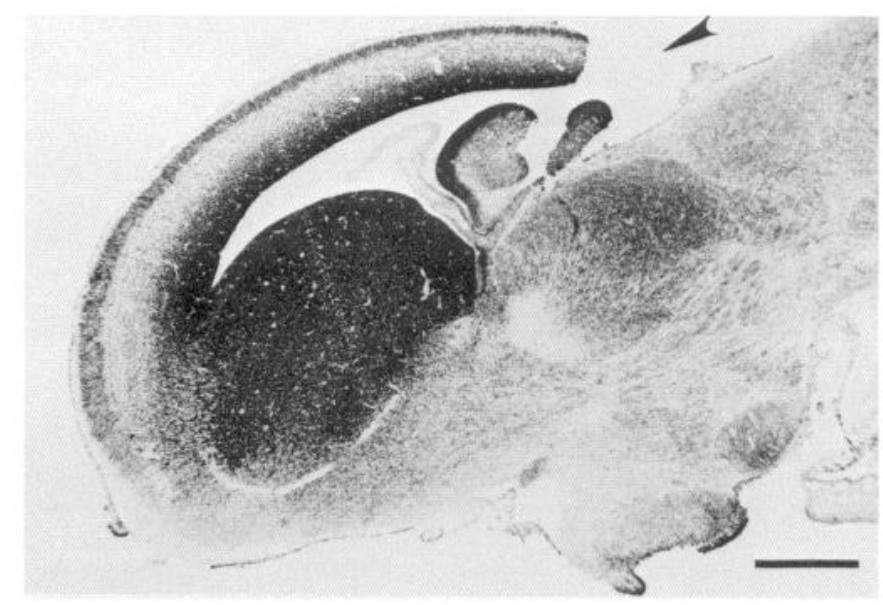

Figure 16. Bright-field photomicrograph of a Nissl-stained sagittal section through the donor brain for the occipital to rostral transplant case illustrated in Figures 17, and 9, $B-D$. The arrowhead marks the region of posterior occipital cortex from which the transplant was taken. Rostral is to the left, dorsal to the top. Scale bar, $0.5 \mathrm{~mm}$.

Usually, the DY-labeled callosal cells were distributed throughout the transplant in a laminar arrangement similar to that seen in the host cortex (see, for example, Fig. 18-color plate), although in a few cases they were found to be preferentially localized within a part of the transplant. In those cases in which the DY injections did not involve the region of cortex homotopic for the transplant, at most a few, or more often, no DY-labeled neurons were found in the transplant. In 20 of the 47 cases with identifiable transplants and successful double-dye injections, DYlabeled callosal neurons and FB-labeled pyramidal tract neurons were found in the transplant. One such case is illustrated in Figures $9, B-D, 16,17$. The region of the occipital cortex removed for transplantation is shown in Figure 16. The location of the transplant in a Nissl-stained section and the corresponding
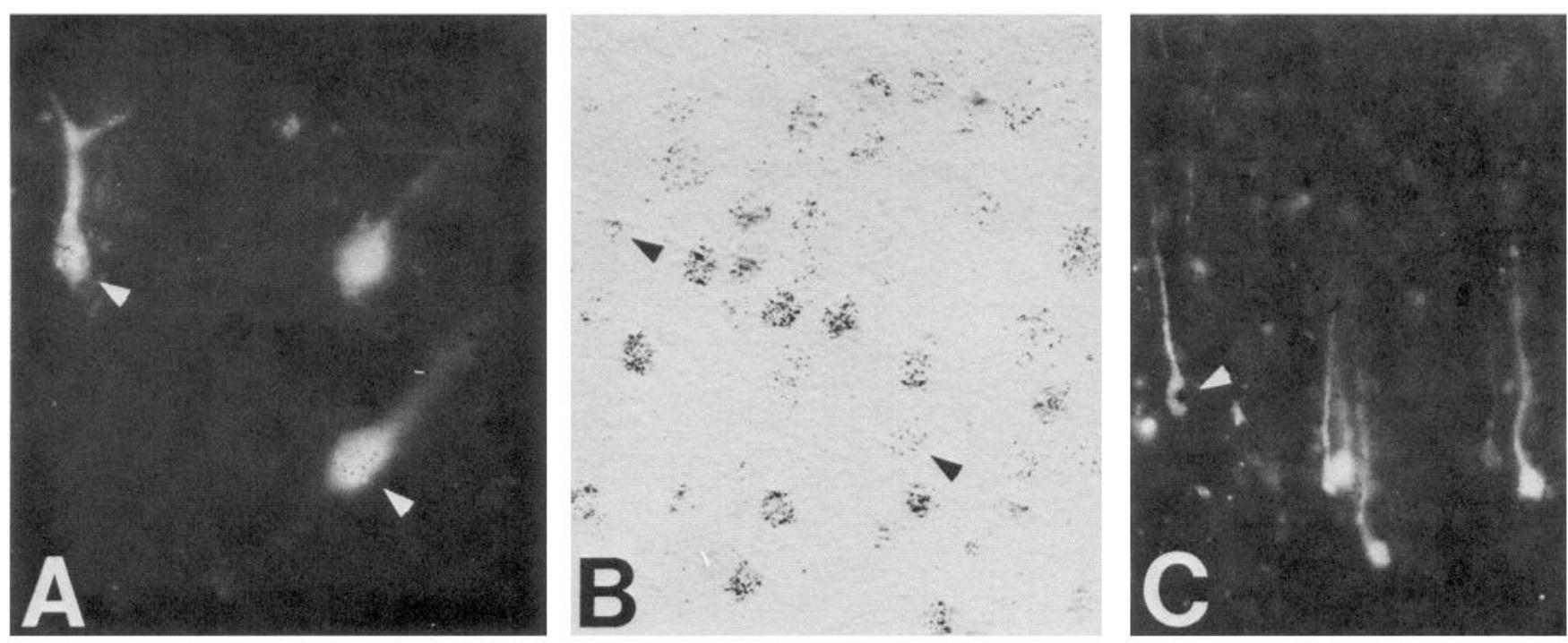

Figure 15. Examples of neurons found within the rostral to occipital transplant of the same case illustrated in Figure 13 that are labeled with both FB and ${ }^{3} \mathrm{H}-\mathrm{TdR}$. $A$ and $B$, Matching fluorescence and bright-field photomicrographs; the arrowheads mark 2 double-labeled neurons. In $A$, the FB labeling as well as the silver grains overlying the nuclei of these 2 neurons can be seen; in $B$, only the silver grains are apparent. $C$, Fluorescence photomicrograph showing another neuron labeled with both FB and ${ }^{3} \mathrm{H}-\mathrm{TdR}$ (arrowhead). Note that the FB-labeled neurons in $A$ and $C$ are pyramidal in form. 

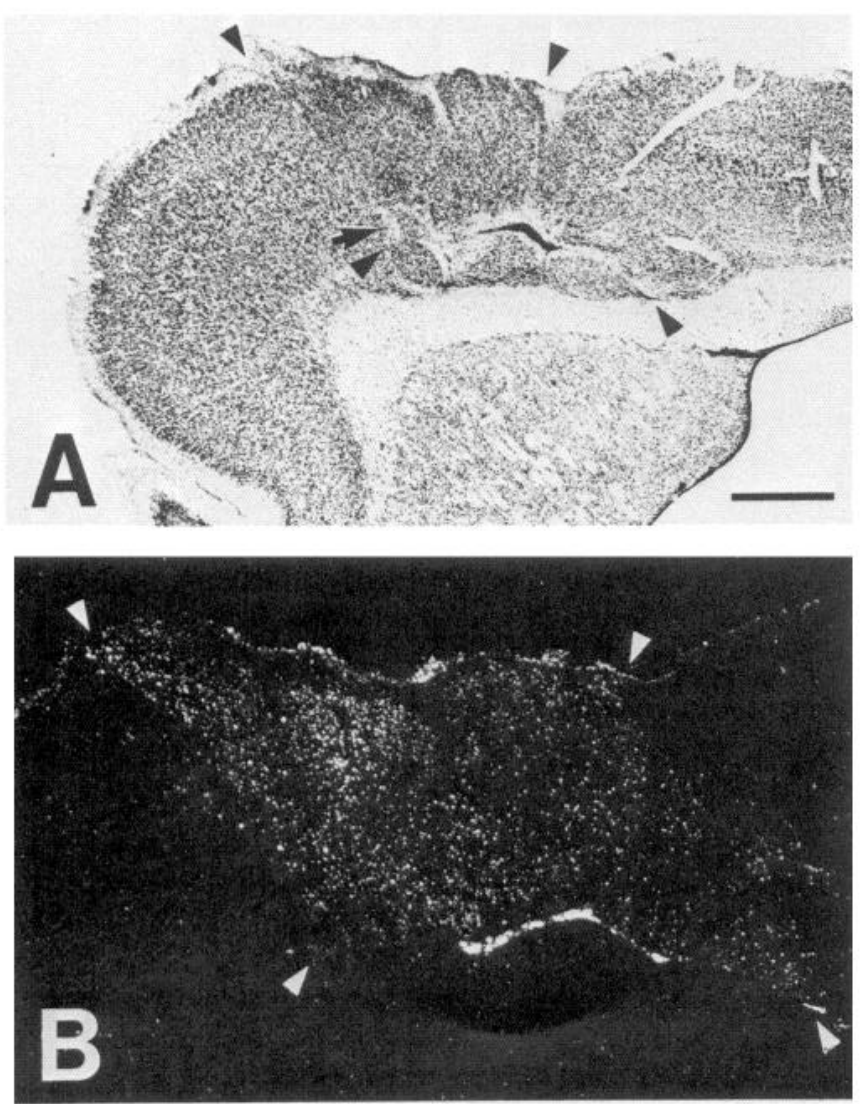

Figure 17. A, Bright-field photomicrograph of a Nissl-stained sagittal section from an occipital to rostral transplant case. $B$, Dark-field photomicrograph of an autoradiogram of the section adjacent to that in $A$. The ${ }^{3} \mathrm{H}$-TdR-labeled transplant can be identified by the clusters of silver grains. The borders of the transplant are indicated with arrowheads. The arrow in $A$ marks the same blood vessel as the arrow in Figure $9 B$. Rostral is to the left, dorsal to the top. Scale bar: $A, 1 \mathrm{~mm} ; B, 0.6 \mathrm{~mm}$.

autoradiogram are shown in Figure 17, $A, B$. The distribution of the fluorescent dye-labeled neurons is similar within both rostral host cortex and the transplanted tissue (Fig. 9B). None of the cells within the transplant in this case, nor in any of the others, is doubly labeled with the 2 fluorescent tracers (see Fig. $9, C, D)$. In every successful case, though, a proportion of the dye-labeled cells was also labeled with ${ }^{3} \mathrm{H}-\mathrm{TdR}$, verifying their donor origin. The results from this paradigm not only indicate that the populations of callosal and pyramidal tract neurons within the transplants are completely separate ones as in normal cortex, but also verify the findings presented above that occipital neurons transplanted to the rostral cortex can develop and maintain pyramidal tract axons.

In the second set of experiments in this group, a piece of ${ }^{3} \mathrm{H}-$ TdR-labeled cortex was removed from a rostral cortical region of an E17 donor brain and transplanted to the posterior occipital region of a newborn. On P34, the superior colliculus was injected with FB and the occipital cortex contralateral to the transplant was multiply injected with DY. The animals were perfused on P40. A transplant was found in 17 of the 24 host rats. DYlabeled neurons were seen in the transplants of 8 of the 12 cases with adequate DY labeling in host cortex, whereas neurons singly labeled with DY and FB were found in the transplants of 4 of the 9 animals in which both injections were successful. Since the findings are very similar to those just described for the

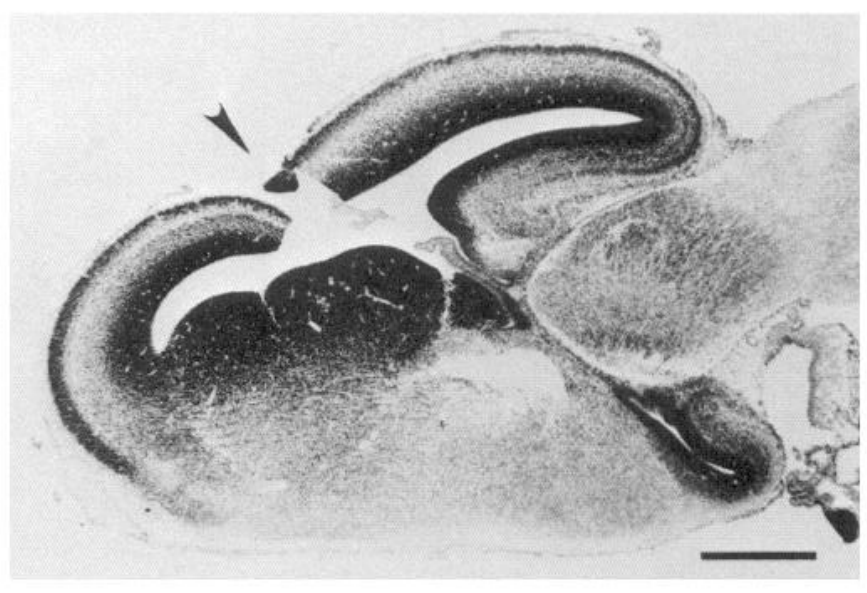

Figure 18. Bright-field photomicrograph of a Nissl-stained sagittal section through the donor brain for the rostral to occipital transplant case illustrated in Figures $9 E, 19,20$. The arrowhead marks the region of cortex from which the transplant was taken. Rostral is to the left, dorsal to the top. Scale bar, $0.5 \mathrm{~mm}$.

preceding paradigm, we will only briefly describe a representative case (illustrated in Figs. 9E, 18-20). The region of rostral cortex removed from the donor brain for transplantation is shown in Figure 18. The transplant, again defined autoradiographically, is located at the posterior pole of the occipital cortex (Fig. 19). Both FB-labeled corticotectal neurons and DY-labeled callosal neurons are found within the transplant (Fig. $9 E$ ). None of the cells within the host occipital cortex, nor within the transplant, of this or any other of the cases, is doubly labeled with the 2 fluorescent dyes. Again, a proportion of the dye-labeled cells in the transplant of this and the other cases is also labeled with ${ }^{3} \mathrm{H}-\mathrm{TdR}$, confirming their donor origin (compare Figs. $9 E$ and 20). As in the similar occipital to rostral transplant cases, when the DY injections were not in the region of the contralateral cortex homotopic for the new locale of the transplant, few, if any, DY-labeled cells were seen in the transplant. Thus, the populations of corticotectal and callosal neurons within the transplants are nonoverlapping, as are pyramidal tract and callosal neurons, suggesting that the transplanted neurons retain their normal neuronal class identities.

Transplantation cavity alone cannot result in an abnormal maintenance of pyramidal tract axons by occipital cortical neurons

As described above, occipital cortical neurons transplanted to a rostral cortical locale extend and maintain pyramidal tract axons. It is possible that this maintenance is due to the removal of a small amount of host rostral cortex to create a cavity in which to place the transplant, and thus slightly reducing the contingent of rostral cortical pyramidal tract neurons. To control for this, we examined the effect of small rostral cortical lesions on the normal restriction of the early, widespread distribution of pyramidal tract neurons (Stanfield et al., 1982). In 9 newborn rats a transplantation cavity was made in rostral cortex in a manner and size similar to that done in the occipital to rostral cases described above, but a piece of donor cortex was not placed in it. The animals were injected with $0.5 \mu \mathrm{l} 2 \%$ FB into the pyramidal decussation on P35 and perfused on P40. The injection was successful and confined to the spinomedullary junction in 5 of the 9 cases. In all 5 of these cases, the distribution of 

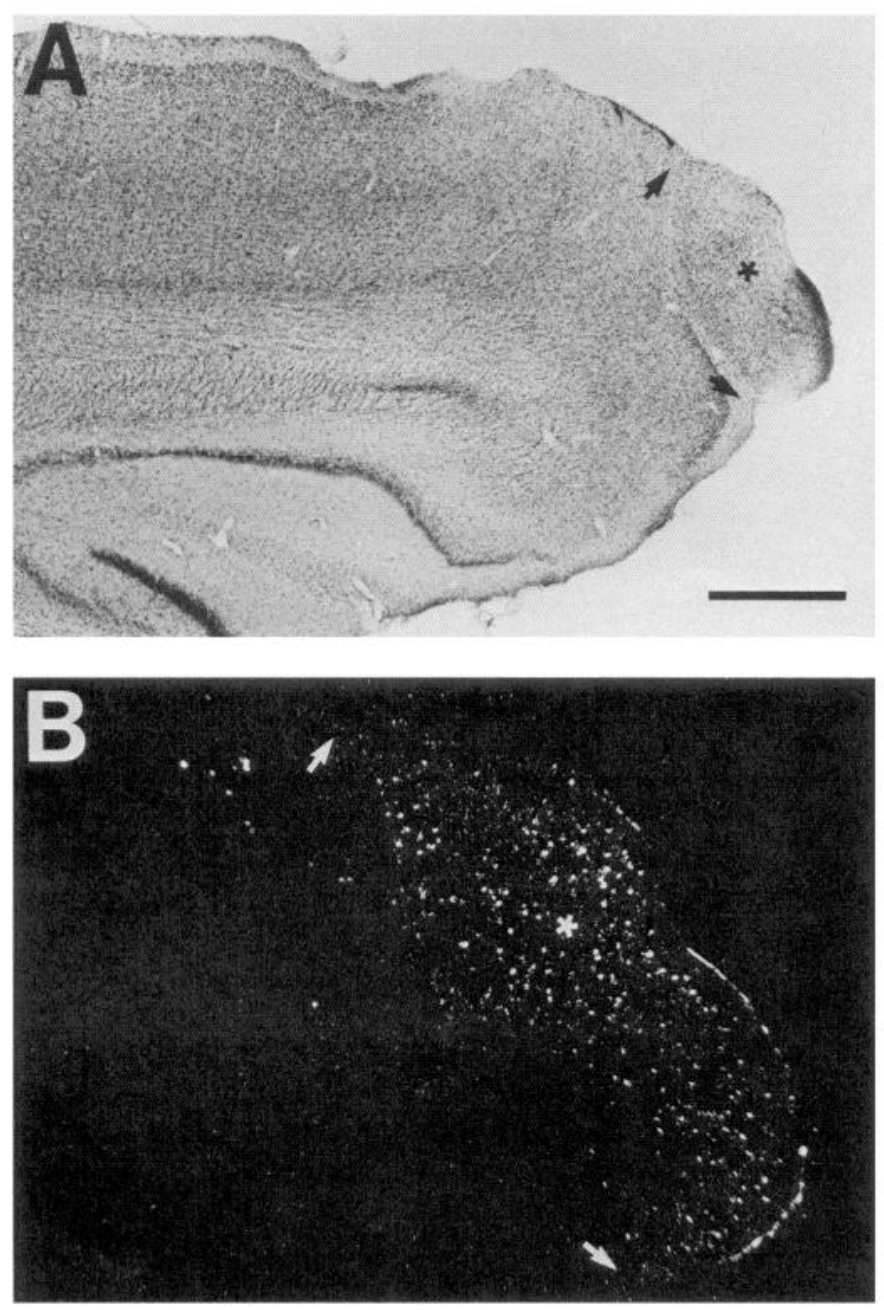

Figure 19. Bright- and dark-field photomicrographs of a Nissl-stained autoradiogram of a sagittal section from a host brain with a rostral to occipital transplant. The transplant border is marked with arrows in both $A$ and $B$. The ${ }^{3} \mathrm{H}$-TdR-labeled transplant can be identified in $B$ by the clusters of silver grains. The region around the asterisk is shown at higher powers in Figures $9 E$ and 20 . Rostral is to the left, dorsal to the top. Scale bar: $A, 750 \mu \mathrm{m} ; B, 350 \mu \mathrm{m}$.

pyramidal tract neurons was virtually identical to that seen in normal adult rats. The labeling pattern of a representative case is shown in Figure 21: a band of well-labeled pyramidal tract neurons is distributed within about the rostral two-thirds of the neocortex and confined to layer $\mathrm{V}$, except in the vicinity of the lesion where the lamination is disrupted to some degree. In this, as well as the other cases, the band of FB-labeled cells comes to an abrupt end near the parietal-occipital border, leaving the caudal third of the neocortex devoid of labeling. Thus, the transplantation technique employed does not by itself lead to an abnormal maintenance of pyramidal tract axons by occipital cortical neurons when left in situ.

\section{Discussion}

The principal finding in our experiments is that a piece of late fetal cortex transplanted to a heterotopic cortical locale in a newborn rat develops a pyramidal tract projection that will be maintained if the transplant is in a region of host cortex that normally maintains a pyramidal tract projection but will be

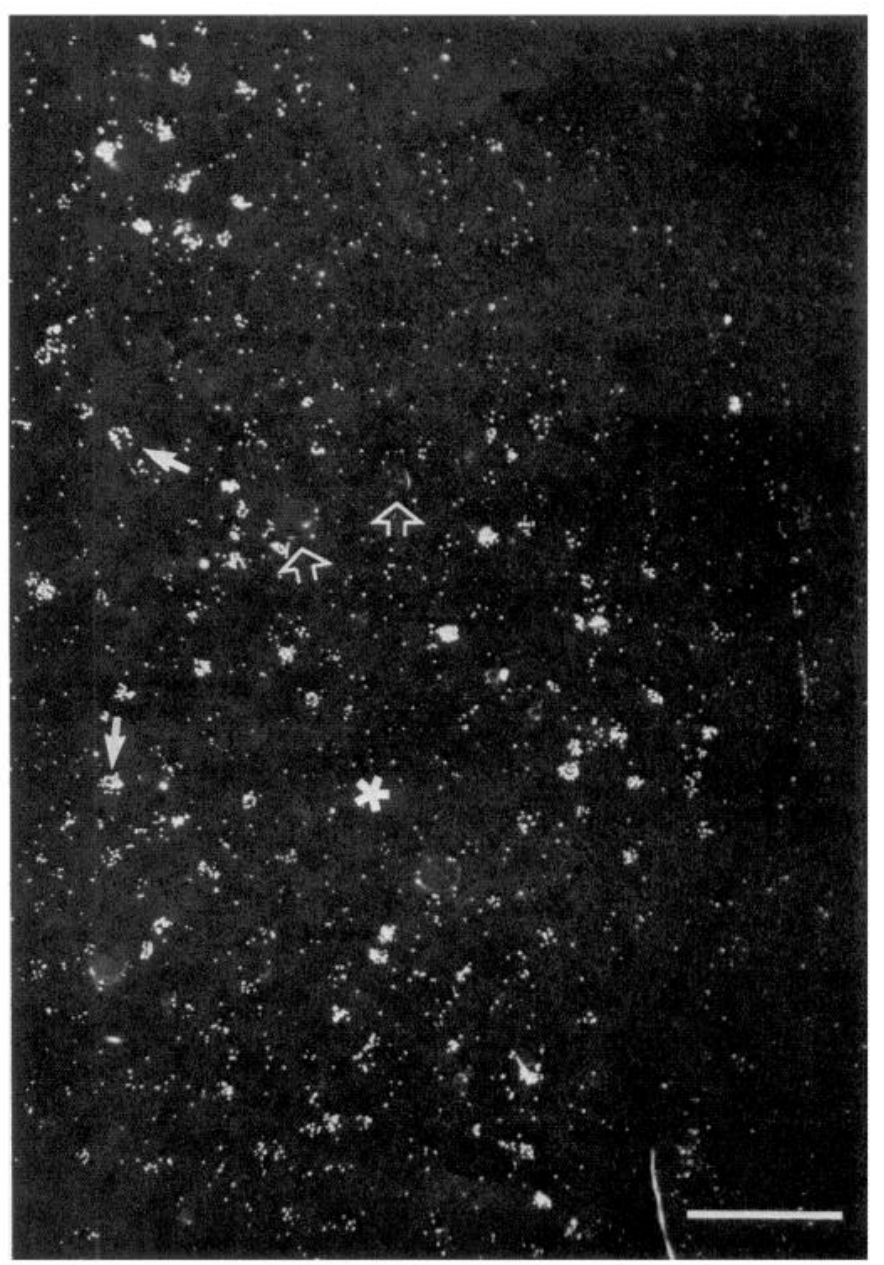

Figure 20. Dark-field photomicrograph of an autoradiogram showing at a higher power the region of the ${ }^{3} \mathrm{H}-\mathrm{TdR}$-labeled transplant marked by the asterisk in Figure 19, A, B. The asterisks are in the same position in the 3 photomicrographs. The solid arrows point to clusters of silver grains over the nuclei of 2 neurons shown in Figure $9 E$ to be also labeled with FB injected into the superior colliculus. The open arrows point to the same blood vessels indicated in Figure $9 E$. Scale bar, $100 \mu \mathrm{m}$.

eliminated if the transplant is in a region of host cortex that normally only transiently sends a projection through the pyramidal tract. Thus, at least in this respect, the transplanted neurons mimic the normal developmental sequence of the projection neurons in the region of cortex to which they are transplanted, rather than of those in the region from where the transplanted neurons originated. An ancillary finding in these experiments is that within the heterotopic transplants, pyramidal tract and corticotectal neurons constitute a separate population from neurons that project callosally, just as they do in the surrounding host cortex and in normal cortex (Catsman-Berrevoets et al., 1980; O'Leary and Stanfield, 1985). This suggests that the set of projections the transplanted neurons develop is characteristic of their neuronal class and is unaltered by their new location, even though this location does influence which subset of projections they will maintain permanently.

The impetus for the series of experiments in this study comes from the demonstrations of the rather homogeneous, widespread distributions of the various projection neuron populations found in developing mammalian neocortex. The early 


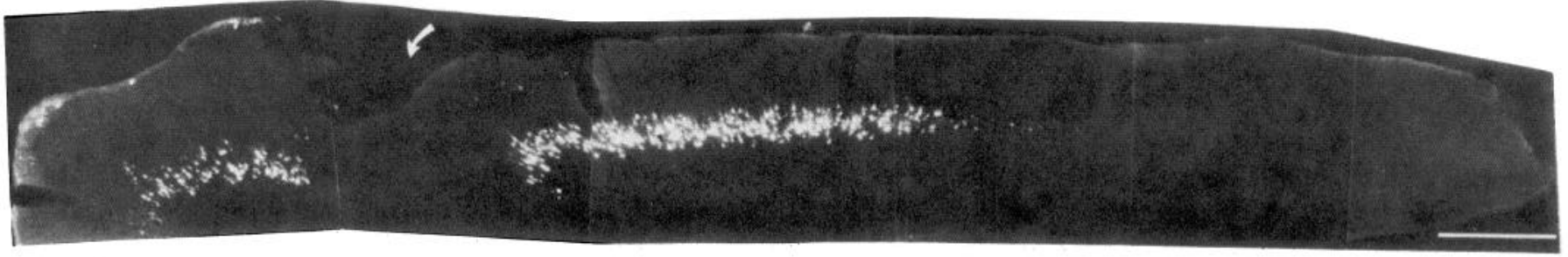

Figure 21. Fluorescence photomontage of a sagittal section of the neocortex of a $\mathbf{P} 40$ rat in which a transplantation cavity was made in rostral cortex but a donor piece of cortex was not inserted. The lesion site is indicated by the arrow. The pyramidal tract neurons labeled by an injection of FB in the pyramidal decussation on P35 are confined to the rostral two-thirds of the neocortex, as in normal rats of this age. Rostral is to the left, dorsal to the top. Scale bar, $1 \mathrm{~mm}$.

distribution of, for example, pyramidal tract neurons in rat cortex is so widespread as to be continuous throughout the neocortical mantle (Stanfield et al., 1982; Bates and Killackey, 1984). In contrast to earlier stages, in adults pyramidal tract neurons are confined to about the rostral two-thirds of the cortex, and none are found within the visual cortex (Stanfield et al., 1982; Miller, 1987; Terashima and O'Leary, 1988). Importantly, this developmental exclusion of pyramidal tract neurons from the visual cortex, as well as from other neocortical areas that do not contain pyramidal tract neurons in the adult, is not the result of cell loss, but rather is due to the selective elimination of the pyramidal tract axons (Stanfield et al., 1982). Many of the early occipital pyramidal tract neurons permanently maintain their early collateral projections to the superior colliculus and/or to the basilar pontine nuclei (O'Leary and Stanfield, 1985) - targets that layer $\mathrm{V}$ neurons in the visual cortex have in common with layer $\mathrm{V}$ projection neurons in more rostral cortical fields. Further, these permanent collaterals are extended before the transient pyramidal tract collaterals are lost (O'Leary and Stanfield, 1985; Stanfield and O'Leary, 1985b). The available evidence suggests that a similar phenomenon occurs in other species of rodents (Crandall et al., 1985; O'Leary and Stanfield, 1986a), and other mammalian orders, including lagomorphs (Distel and Hollander, 1980), and marsupials (Cabana and Martin, 1984). Interestingly, there seemed to be marked species differences in how far caudal the transient pyramidal tract axons descend during development before they are eliminated. For example, while some transient occipital pyramidal tract axons in rat enter the spinal cord and a few extend as far as lumbar levels (Stanfield and O'Leary, 1985b), in rabbit most do not appear to reach the spinomedullary junction (Distel and Hollander, 1980).

Similarly, the patchy and limited presence of callosally projecting neurons in the adult neocortex is sculpted out of an initially widespread distribution through a process involving the selective loss of callosal axon collaterals without the concomitant death of the parent neurons (Innocenti, 1981; O'Leary et al., 1981), many of which retain an ipsilateral corticocortical projection (Ivy and Killackey, 1982; Innocenti et al., 1986). Early widespread distributions of callosally projecting neurons have now been reported to be present in every region of the developing neocortex and in every mammalian order examined, including rodents (Ivy et al., 1979; Olavarria and van Sluyters, 1985), lagomorphs (Chow et al., 1981), marsupials (i.e., neocortical commissural neurons; Cabana and Martin, 1985), carnivores (Innocenti et al., 1977; Innocenti and Caminiti, 1980; Feng and Brugge, 1983; Cornwell et al., 1984), and primates (Killackey and Chalupa, 1986; Dehay et al., 1988). A comparable sequence of developmental events, beginning with a wide- spread distribution of a class of projection neuron and culminating with the selective elimination of certain collateral projections formed by members of that class residing in specific regions of cortex, also appears to bring about the limited distributions of corticotectal (Thong and Dreher, 1986) and ipsilateral corticocortical neurons (Dehay et al., 1984; Innocenti and Clarke, 1984; Price and Blakemore, 1985; Clarke and Innocenti, 1986) seen in adult mammals. It should be mentioned that a few retrograde tracing studies of the development of certain cortical projections have failed to identify as widespread of an early distribution of projection neurons as seen in the aforementioned studies (Dehay et al., 1988; Schreyer and Jones, 1988 - but see O'Leary et al., 1988). While some of these differences may be due to technical considerations, such as the tracer used (see Ivy et al., 1984) or the period of development examined, they may as well reflect the fact that, in some instances, transient axon collaterals are eliminated before they attain the target region of the relevant fiber system. As noted above, the extent of growth that transient collaterals attain within a given system even varies among species (see O'Leary and Stanfield, 1986a). Thus, the available evidence indicates that in many, if not all, cortical projection systems the restricted patterns of the projection neurons characteristic of the adult neocortex do not arise de novo, but rather emerge during development from an initially widespread distribution through the loss of axon collaterals.

These observations suggest that the same complement of projection neuron classes is present throughout the neocortex at early stages and that factors which differ regionally influence the subsequent elimination of those initially extended projections that may be inappropriate and the retention of those projections that are appropriate for the functional role of a given cortical locale. A test of this possibility would be to transplant newly generated cortical neurons, allow them to mature in a heterotopic cortical locale, and then determine if they behave, in terms of their maintained projections, like those neurons in their region of origin or like the host neurons in their new locale. The transplantation technique has proven to be a useful tool of experimental embryology since the early part of this century. However, the application of this technique to the study of the development of the CNS has largely been limited to nonmammalian species. Even the recent surge of interest in the transplantation of mammalian CNS tissue has, with a few exceptions, focused on aspects of the viability of the transplant and its ability to form connections with the host brain, rather than on what influence a new environment may have on cell-type-specific characteristics of the transplanted neurons. In our present experiments, as well as in our previous study (Stanfield and O'Leary, 
1985a), we have taken advantage of the fact that pieces of fetal rat brain survive well when transplanted into young donor brains to examine the relative influence of site of origin and final locale on the projections which are initially extended and those which are eventually maintained by cortical neurons which are transplanted, heterotopically, to a new region of cortex during development.

In every instance, both in our previous study (Stanfield and O'Leary, 1985a), as well as in the present series of experiments, the projections maintained by the heterotopic cortical transplants are those appropriate to the new cortical locale. In cases in which pieces of fetal occipital cortex had been placed in a rostral locale, many of the transplanted neurons are labeled with FB injected into the pyramidal decussation on P34; however, in animals in which pieces of fetal rostral cortex are transplanted to an occipital locale, when $\mathrm{FB}$ is injected into the pyramidal decussation on P34, no retrogradely labeled cells are found in the transplant, even though ncurons in these transplants had extended long axonal projections as evidenced by the fact that many cells within the transplants can be labeled by injections of DY into the superior colliculus, a collateral target of developing pyramidal tract neurons in both occipital and rostral cortex. As in rostral cortex, callosally projecting neurons in the occipital cortex are only labeled within the heterotopic transplants when the injections of the retrograde tracer into the contralateral hemisphere also label cells within the adjacent host cortex. Thus, in this transplant paradigm as well, the transplanted neurons maintain projections appropriate to their new locale rather than to their region of origin.

Furthermore, our observations suggest that the selective elimination of axon collaterals is involved in the determination of the maintained targets of the transplanted neurons, just as it is involved in the determination of the maintained targets of the host cortex. When an animal that had received a rostral to occipital transplant was injected with FB in the pyramidal decussation several days after the transplantation, but at an early stage when the normal, transient occipital cortical component of the pyramidal tract was still present, labeled neurons were seen within the transplant, as well as within the adjacent host occipital cortex. Yet, as just described, no pyramidal tract neurons were found within such transplants at ages by which the normal occipital cortical component of the pyramidal tract is eliminated but a sizable population of corticotectal neurons is present. Thus, not only do heterotopic fetal cortical transplants maintain projections appropriate to their new locale, they even seem to extend and then eliminate the same transient projections extended and eliminated by that region of host cortex.

These observations are consistent with the idea that the heterotopic cortical transplants contain the same complement of projection neuron classes as found in the host cortex of the new locale and that the similarity between the projections established by the transplants and those of the adjacent cortex is due to the fact that the various projection neuron classes within the transplants respond in the same manner as host neurons of the same classes within that region; that is, the neurons are expressing projections both appropriate for their new locale and characteristic of their neuronal class. If this were true, we should expect that the transplanted occipital neurons that extend a pyramidal tract axon when in the rostral cortex are the same cells that would have transiently extended a pyramidal tract projection had they remained in the occipital cortex. While we do not have direct evidence for this, several observations suggest that this is so. First, the fact that some of the dye-labeled cells found within the transplants arc doubly labclcd with ${ }^{3} \mathrm{H}-\mathrm{TdR}$ (to which the donor animals were exposed on E15) indicates that at least some of the transplanted neurons that could be retrogradely labeled from the pyramidal decussation were generated at a time appropriate for layer $\mathrm{V}$ neurons in general (Bruckner et al., 1976), and pyramidal tract neurons in particular (D. D. M. O'Leary and B. B. Stanfield, unpublished observations). Second, in cases in which the normal cortical lamination of the transplant was to some degree retained, the cells labeled with FB injected into the pyramidal decussation were confined to a band that, in terms of the Nissl-stained appearance of the neurons it contained and the overall appearance of the transplant, seemed to correspond to layer $\mathrm{V}$; whereas neurons labeled with DY injected into the contralateral cortex were commonly distributed throughout the cellular layers of the transplant, as in normal cortex. Third, cells well labeled with FB (thus making their shape discernable) had pyramidal-like morphologics (scc Figs. 5, $9 C$, 15) rather than one of the many distinctive morphologies characteristic of other cortical cell types. Finally, in the transplants, as in layer $\mathrm{V}$ of normal rat occipital cortex (O'Leary and Stanfield, 1985), neurons with subcortical projections, either to the superior colliculus or through the pyramidal tract, and callosally projecting neurons constitute completely separate projection neuron populations, suggesting that these distinct projection neuron populations retain, in the transplants, their normal identities. Thus, although we cannot conclude with complete certainty, these observations do suggest that the transplanted neurons extend the same projections that they would have if left in situ. Our findings are consistent with the interpretation that the projections extended by the transplanted neurons are appropriate for the class of cortical projection neuron to which they belong and that the new locale influences the normally occurring process of selective collateral elimination resulting in the maintenance of projections that are appropriate for the new region.

While heterotopic transplantation is a useful technique with which to approach the issue of position related influences on maintained projections, our approach is not without problems. Before we address more specific concerns, it may be important to consider at least one of the more general issues that may be particularly relevant: that is, whether the transplantation procedure might somehow alter the character of the transplanted neurons, so as to strip them of their identities, and that these cells then assume identities appropriate to the locale in which they find themselves. We feel that this is unlikely for several reasons. First, young postmitotic cortical neurons show a remarkable capacity to retain many aspects of their identity even when they are microinjected into developing cortex as a dissociated cell suspension and in few numbers (McConnell, 1985, 1988). Our own procedure of transplanting an intact piece of cortex would seem to present much less of a challenge to the neuronal identity of the young postmitotic neurons. We should emphasize here, especially since the final mitotic cycle may be critical in determining the neuronal identity of a cell, that many of the cortical neurons within our transplants, including most if not all of the layer $\mathrm{V}$ neurons, are postmitotic at the time of transplantation (Bruckner et al., 1976). And, judging from the large numbers of neurons heavily labeled with ${ }^{3} \mathrm{H}-\mathrm{TdR}$ that are found in the transplants at P13 and P40, many of the neurons within our transplants had undergone their final round of DNA synthesis as much as $2 \mathrm{~d}$ before transplantation. Second, transplantation of fetal neurons does not seem to alter the neuronal 
identity of the cells dramatically, since when placed in entirely different regions of the brain, transplanted neurons still show target selectivity characteristic of their region of origin (Alvarado-Mallart and Sotelo, 1982). This seems to be true even among markedly different regions within the cerebral cortex. In some of our experiments, a piece of the hippocampal formation was left attached to the piece of fetal posterior neocortex that was transplanted to a rostral cortical locale. Although neurons within the neocortical part of the transplant are labeled following injections of FB into the pyramidal decussation and DY into the contralateral neocortex, none of the cells in the transplanted hippocampal tissue (which could be readily identified by its characteristic single layer of densely packed pyramidal cells) was ever found to be labeled (O'Leary and Stanfield, unpublished observations). Finally, the observations detailed above which suggest that the projection neurons within the transplants develop projections appropriate for the neuronal class they were destined to be a member of if left in situ also argue that these cells had retained their identity during transplantation.

An aspect of our methodology that may be of concern is that, in addition to being heterotopic, our transplants were also slightly heterochronic; that is, the pieces of donor cortex were taken at $\mathrm{E} 17$ and placed into brains of $\mathrm{PO}$ hosts. While we would have liked to have made isochronic transplants, we thought it important to take the donor tissue at the age chosen not only because fetal brain tissue responds best to transplantation, but also becausc we wanted the transplanted layer $\mathrm{V}$ neurons to be postmitotic and yet not have extended axons for any great distance prior to transplantation, and since there would be great technical difficulties associated with using E17 hosts, we settled on placing the transplants into newborn hosts. Our results indicate that the fact that the transplanted neurons in our experiments were about 4-5 d younger than the neurons in the adjacent host cortex did not prevent them from establishing ample connections, and thus it would seem that this aspect of our experiments should be of minimal concern.

In our earlier study, we tested for the presence of pyramidal tract neurons within occipital to rostral transplants by injecting FB into the pyramidal decussation on P25 (Stanfield and O'Leary, 1985a). A potential concern with regard to this previous finding is that the transplantation procedure slows the normal maturation of the transplanted neurons so that the projections present at P25 might include some that will not be maintained. To confirm that occipital neurons transplanted to a rostral cortical location do permanently maintain a pyramidal tract projection, we repeated in the present study our original transplantation paradigm with the change to using $\mathrm{P} 40$ as the end point of the experiments following tracer injections on P34. Since in normal rats the restricted adult distribution of pyramidal tract neurons is established no later than P17 (Stanfield et al., 1982; Stanfield and O'Leary, 1985b), this allows for a substantial temporal buffer. The fact that we have been able to physiologically identify pyramidal tract neurons in occipital to rostral transplants in rats of 3 months to 1 year of age (Porter et al., 1987) strongly supports our belief that the pyramidal tract axons extended by neurons in such transplants are permanent. However, the most direct evidence that by P34 the transplanted neurons in our experiments had matured beyond the stage during which they would undergo selective collateral elimination comes from our observation that while many neurons within rostral to occipital transplants have pyramidal tract collaterals on P9, by P34 all of these have been eliminated. Thus, it seems that any retardation of the normal rate of maturation of neurons within the transplanted cortex that may occur does not present a problem to the interpretation of our experiments.

A problem not unique to our transplantation experiments, but one that is important to discuss in the context of our findings, in particular those from our occipital to rostral paradigm, is the necessity of removing some host cortex in order to create a site for the transplant. Of potential concern is that occipital neurons transplanted to a rostral cortical locale permanently retain pyramidal tract axons as a direct result of the removal of some host pyramidal tract neurons making available terminal space within the targets of pyramidal tract neurons. Were this true, one might predict that if a transplant cavity is made, but no transplant is placed in it, some occipital cortical neurons would retain the pyramidal tract axons that they normally extend transiently into the lower medulla and spinal cord (Stanfield et al., 1982; Stanfield and O'Leary, 1985). We find, though, that the occipital cortical neurons still lose their pyramidal tract axons under such conditions. Perhaps even more compelling is the fact that neurons within the occipital to rostral transplants extend and maintain pyramidal tract axons, while in situ occipital neurons in these same hosts-neurons which at the time the transplantation is done have already extended axons well into the medullary pyramidal tract-develop but lose this projection. In this paradigm, one might expect that the transient pyramidal tract axons of host occipital neurons, given the fact that they already have extended through the pyramidal tract at the time the transplantations are done (Stanfield and O'Leary, 1985), would have a competitive advantage over the transplanted neurons and would be preferentially retained, but they are not. When considered together, these observations strongly argue that position within the tangential plane of the cortex (and factors associated with position) is a decisive influence in the retention or elimination of pyramidal tract axon collaterals.

A final technical consideration is the possibility that some of the donor pyramidal tract neurons were axotomized at the time of the transplanting and subsequently reinitiated the elongation of their primary axon. Our observation that some of the dyelabeled cells were generated at least $2 \mathrm{~d}$ prior to transplantation indicates that these may have had sufficient time to extend an axon out of the region of cortex that was taken for transplantation. Anterograde labeling studies do indicate that by E17 some corticofugal axons have grown a short distance out of the neocortex and into the internal capsule (Schreyer and Jones, 1982), and there is some evidence to suggest that early pyramidal tract axons can regenerate (Kalil and Reh, 1982; but see Tolbert and Der, 1987). However, it seems that any regrowth of cut axons that might have occurred in our experiments would complicate the interpretation of our results only had we found that the heterotopically transplanted neurons maintain the projections characteristic of their original locale; in fact, we have found just the opposite.

During recent years it has been argued that, from a developmental point of view, the neocortex can be considered an ensemble of roughly parallel, radial ontogenetic columns (Rakic, 1978,1981 ). Each of these columns comprises neurons that are of different classes but that arise from the same proliferative unit within the ventricular zone. Subsequent to their proliferation, these neurons become vertically disposed as a result of their migration along the radial glial scaffolding. Rakic (1978, $1981,1988)$ has argued that the proliferative units within the ventricular zone constitute a "proto-map" of the prospective 
cytoarchitectonic areas and that neuronal types produced within proliferative units vary from region to region. Our results do not directly contradict such a prespecification of regional differences within the cortical ventricular zone; however, they do demonstrate that any such regional prespecification of cortical neurons cannot be rigid and the young neurons remain, in terms of their projections, malleable. Our findings, though, are consistent with an alternative hypothesis that the proliferative units throughout the neocortical neuroepithelium give rise to radial ontogenetic columns that, aside from their tangential position, are initially indistinguishable in terms of their complement of neuronal classes. The experiments which indicate that the maintained projections of heterotopically transplanted pieces of fetal cortex are appropriate for the transplants' new cortical locale, that the maintained projections of both heterotopic transplants and regions of normal cortex emerge during development through a process involving the selective elimination of specific branches of initially more widely targeted collateral projections, and the observations suggesting that the transplanted neurons maintain their initial identity as to the class of projection neuron they belong are all consistent with this concept.

We propose that equivalent proliferative units present throughout the neocortical mantle produce comparable lineages of neurons that comprise the full complement of classes of cortical neurons. The subsequent radial migration of the generated neurons brings homologous neuronal classes in register to form the cytoarchitectonic layers of the mature cortex. Initially, cells throughout the cortex that are of the same projection neuron type extend similar projections. The acquisition of the marked regional differences in cortical projections is a subsequent event that is achieved largely through a process of selective collateral elimination, although other processes, such as cell death (Finlay and Slattery, 1983; Price and Blakemore, 1985; but also see Crandall et al., 1985), may contribute as well to the cstablishment of connectional and other regional differences. According to this schema, the generation of the elaborate regional differentiation found in the mature cortex would not depend on any regional prespecification within the neocortical neuroepithelium. Thus, the existence of equivalent proliferative units could markedly simplify the early development of the cortex and should as well introduce the potential for considerable cortical plasticity, both during development and during phylogeny; as it may be a relatively simple matter either to alter the allocation of proliferative units to match, for example, changes in the demands of the periphery or to increase at an early stage the total number of proliferative units initially available. It would be of considerable interest to better define these initial proliferative units in terms of the number of precursor cells they contain and the lineage of the cells they produce.

\section{References}

Alvarado-Mallart, R. M., and C. Sotelo (1982) Differentiation of cerebellar anlage heterotopically transplanted top adult rat brain: A light and electron microscopic study. J. Comp. Neurol. 212: 247-267.

Bates, C. A., and H. P. Killackey (1984) The emergence of a discretely distributed pattern of corticospinal projection neurons. Dev. Brain Res. 13: 265-273.

Bentivoglio, M., J. G. J. M. Kuypers, C. E. Catsman-Berrevoets, H. Loewe, and $O$. $O$. Dann (1980) Two new fluorescent retrograde neuronal tracers which are transported over long distances. Neurosci. Lett. 18: 25-30.

Bruckner, G., V. Mares, and D. Bicsold (1976) Ncurogenesis in the visual system of the rat. An autoradiographic investigation. J. Comp. Neurol. 166: 245-256.

Cabana, T., and G. F. Martin (1984) Developmental sequence in the origin of descending spinal pathways. Studies using retrograde transport techniques in the North American opossum (Didelphus virginiana). Dev. Brain Res. 15: 247-263.

Cabana, T., and G. F. Martin (1985) The development of commissural connections of somatic motor-sensory areas of neocortex in the North American opossum. Anat. Embryol. 17: 121-128.

Castro, A. J., N. Tonder, N. A. Sunde, and J. Zimmer (1987) Fetal cortical transplants in the cerebral hemisphere of newborn rats: A retrograde fluorescence analysis of connections. Exp. Brain Res. 66 . $533-542$.

Catsman-Berrevoets, C. E., R. M. Lemon, C. A. Verburgh, M. Bentivoglio, and H. G. J. M. Kuypers (1980) Absence of callosal collaterals derived from rat corticospinal neurons. A study using fluorescent retrograde tracing and electrophysiological techniques. Exp. Brain Res. 39: $433-440$.

Chang, F.-L. F., J. G. Steedman, and R. D. Lund (1984) Embryonic cerebral cortex placed in the occipital region of newborn rats makes connections with host brain. Dev. Brain Res. 13: 164-166.

Chang, F.-L. F., J. G. Steedman, and R. D. Lund (1986) The lamination and connectivity of embryonic cerebral cortex transplanted into newborn rat cortex. J. Comp. Neurol. 244: 401-411.

Chow, K. K., H. D. Baumbach, and R. Lawson (1981) Callosal projections of the striate cortex in the neonatal rabbit. Exp. Brain Res. 42: $122-126$

Clarke, S., and G. M. Innocenti (1986) Organization of immature intrahemispheric connections. J. Comp. Neurol. 251: 1-22.

Cornwell, P., R. Ravizza, and B. Payne (1984) Extrinsic visual and auditory connections in the 4-day-old kitten. J. Comp. Neurol. 229: 97-120.

Cowan, W. M., D. I. Gottlieb, A. E. IIendrickson, J. L. Price, and T. A. Woolsey (1972) The autoradiographic demonstration of axonal connections in the central nervous system. Brain Res. 37: 21-51.

Crandall, J. E., J. M. Whitcomb, and V. S. Caviness, Jr. (1985) Development of the spino-medullary projection from the mouse barrel field. J. Comp. Neurol. 239: 205-215.

Dehay, C., J. Bullier, and H. Kennedy (1984) Transient projections from the fronto-parietal and temporal cortex to areas 17,18, and 19 in the kitten. Exp. Brain Res. 57: 208-212.

Dehay, C., H. Kennedy, J. Bullier, and M. Berland (1988) Absence of interhemispheric connections of area 17 during development in the monkey. Nature 331: 348-350.

Distel, H., and H. Hollander (1980) Autoradiographic tracing of developing subcortical projections of the occipital region in fetal rabbits. J. Comp. Neurol. 192: 505-518.

Feng, J. Z., and J. F. Brugge (1983) Postnatal development of auditory callosal connections in the kitten. J. Comp. Neurol. 214: 416-426.

Finlay, G. L., and M. Slattery (1983) Local differences in amount of early cell death in neocortex predict adult local specializations. Science 219: 1349-1351.

Floeter, M. K., and E. G. Jones (1985) Transplantation of fetal postmitotic neurons to rat cortex: Survival, early pathway choices and long-term projections of outgrowing axons. Dev. Brain Res. 22: 1938.

Herman, J. P., N. Abrous, A. Vigny, J. Dulluc, and M. Le Moal (1988) Distorted development of intracerebral grafts: Long-term maintenance of tyrosine hydroxylase-containing neurons in grafts of cortical tissue. Dev. Brain Res. 40: 81-88.

Innocenti, G. M. (1981) Growth and reshaping of axons in the establishment of visual callosal connections. Science 212: 824-827.

Innocenti, G. M., and R. Caminiti (1980) Postnatal shaping of callosal connections from sensory areas. Exp. Brain Res. 38: 381-394.

Innocenti, G. M., and S. Clarke (1984) Bilateral transitory projection to visual areas from auditory cortex in kittens. Dev. Brain Res. 14: $143-148$.

Innocenti, G. M., L. Fiore, and R. Caminiti (1977) Exuberant projection into the corpus callosum from the visual cortex of newborn cats. Neurosci. Lett. 4: 237-242.

Innocenti, G. M., S. Clarke, and R. Kraftsik (1986) Interchange of callosal and association projections in the developing visual cortex. J. Neurosci. 6: 1384-1409.

Ivy, G. O., and H. P. Killackey (1982) Ontogenetic changes in the projections of neocortical neurons. J. Neurosci. 2: 735-743. 
Ivy, G. O., R. M. Akers, and H. P. Killackey (1979) Differential distribution of callosal projection neuons in the neonatal and adult rat. Brain Res. 173: 532-537.

Ivy, G. O., H. J. Gould III, and H. P. Killackey (1984) Variability in the distribution of callosal projection neurons in the adult rat parietal cortex. Brain Res. 306: 53-61.

Jaeger, C. B., and R. D. Lund (1980) Transplantation of embryonic occipital cortex to the brain of newborn rats: A golgi study of mature and developing transplants. J. Comp. Neurol, 200: 213-230.

Jaeger, C. B., and R. D. Lund (1981) Transplantation of embryonic occipital cortex to the brain of newborn rats. An autoradiographic study of transplant histogenesis. Exp. Brain Res. 40: 265-272.

Kalil, K., and T. Reh (1982) A light and electron microscopic study of regrowing pyramidal tract fibers. J. Comp. Neurol. 211: 265-275.

Keizer, K., H. G. J. M. Kuypers, A. M. Huisman, and O. Dann (1983) Diamidino yellow dihydrochloride (DY-2HCl); a new fluorescent retrograde neuronal tracer which migrates only very slowly out of the cell. Exp. Brain Res. 51: 179-191.

Killackey, H. P., and L. M. Chalupa (1986) Ontogenetic changes in the distribution of callosal projection neurons in the postcentral gyrus of the fetal rhesus monkey. J. Comp. Neurol. 244: 331-348.

Koester, S. E., and D. D. M. O'Leary (1989) Selective axon growth not axon loss produces connectional distinctions between callosal and subcortically projecting layer 5 neurons. Soc. Neurosci. Abstr. (in press).

Lund, R. D., and M. J. Mustari (1977) Developmental of the geniculocortical pathway in rats. J. Comp. Neurol. 173: 289-306.

Lund, R. D., K. Rao, M. H. Hankin, H. W. Kunz, and T. J. Gill, III (1978) Transplantation of retina and visual cortex to rat brains of different ages. Maturation, connection patterns, and immunological consequences. Ann. NY Acad. Sci. 494: 227-241.

McConnell, S. K. (1985) Migration and differentiation of cerebral cortical neurons after transplantation into the brains of ferrets. Science 229: $1268-1271$.

McConnell, S. K. (1988) Fates of visual cortical neurons in the ferret after isochronic and hetcrochronic transplantation. J. Ncurosci. 8: 945-974.

Miller, M. W. (1987) The origin of corticospinal projection neurons in rat. Exp. Brain Res. 67: 339-351.

Olavarria, J., and R. van Sluyters (1982) The projection from striate and extrastriate cortical areas to the superior colliculus in the rat. Brain Res. 242: 332-336.

Olavarria, J., and R. van Sluyters (1985) Organization and postnatal development of callosal connections in the visual cortex of the rat. J. Comp. Neurol. 239: 1-26.

O'Leary, D. D. M., and B. B. Stanfield (1985) Occipital cortical neurons with transient pyramidal tract axons extend and maintain collaterals to subcortical but not intracortical targets. Brain Res. 336: 326-333.

O'Leary, D. D. M., and B. B. Stanfield (1986a) A transient pyramidal tract projection from the visual cortex in the hamster and its removal by selective collateral elimination. Dev. Brain Res. 27: 87-99.

O'Leary, D. D. M., and B. B. Stanfield (1986b) Heterotopic transplants of visual and sensorimotor cortex develop and maintain projections characteristic of new cortical locale. Anat. Rec. 214: 93A.
O'Leary, D. D. M., and B. B. Stanfield (1986c) Maintained projections of heterotopic transplants of fetal visual and sensorimotor cortex are characteristic of new cortical locale. Soc. Neurosci. Abstr. 12: 1049.

O'Leary, D. D. M., B. B. Stanfield, and W. M. Cowan (1981) Evidence that the early postnatal restriction of the cells of origin of the callosal projection is due to the elimination of axonal collaterals rather than to the death of neurons. Dev. Brain Res. 1: 607-617.

O'Leary, D. D. M., T. Terashima, B. L. Schlaggar, and B. B. Stanfield (1988) Demonstration of corticospinal neurons in primary visual cortex of early postnatal rats. Anat. Rec. 220:72A.

Porter, L. L., J. M. Cedarbaum, D. D. M. O'Leary, B. B. Stanfield, and H. Asanuma (1987) The physiological identification of pyramidal tract neurons within transplants in the rostral cortex taken from the occipital cortex during development. Brain Res. 436: 136-142.

Price, D. J., and C. Blakemore (1985) The postnatal development of the association projection from visual cortical area 17 to area 18 in the cat. J. Neurosci. 5: 2443-2452.

Rakic, P. (1978) Neuronal migration and contact guidance in primate telencephalon. Postgrad. Med. J. 54: 25-40.

Rakic, P. (1981) Developmental events leading to laminar and areal organization of the neocortex. In The Organization of the Cerebral Cortex, F. O. Schmitt, F. G. Worden, F. G. Adelman, and S. G. Dennis, eds., pp. 7-28, MIT Press, Cambridge, MA.

Rakic, P. (1988) Specification of cerebral cortical areas. Science 241 $170-176$.

Rogers, A. W. (1973) Techniques of Autoradiography, Elsevier, Amsterdam.

Schreyer, D. J., and E. G. Jones (1982) Growth and target finding by axons of the corticospinal tract in the prenatal and postnatal rats. Neuroscience 7: 1837-1853.

Schreyer, D. J., and E. G. Jones (1988) Topographic sequence of outgrowth of corticospinal axons in the rat: A study using retrograde axonal labeling with Fast blue. Dev. Brain Res. 38: 89-101.

Stanfield, B. B., and D. D. M. O'Leary (1985a) Fetal occipital cortical neurons transplanted to the rostral cortex can extend and maintain a pyramidal tract axon. Nature 313: 135-137.

Stanfield, B. B., and D. D. M. O'Leary (1985b) The transient corticospinal projection from the occipital cortex during the postnatal development of the rat. J. Comp. Neurol. 238: 236-248.

Stanfield, B. B., D. D. M. O'Leary, and C. Fricks (1982) Selective collateral elimination in early postnatal development restricts cortical distribution of rat pyramidal tract neurones. Nature 298: 371-373.

Terashima, T., and D. D. M. O'Leary (1988) Quantitative analysis of the cortical distribution of pyramidal tract neurons in young and adult rats. Anat. Rec. 220: 96A.

Thong, I. G., and B. Dreher (1986) The development of the corticotectal pathway in the albino rat. Dev. Brain Res. 25: 227-238.

Tolbert, D. L., and T. Der (1987) Redirected growth of pyramidal tract axons following neonatal pyramidotomy in cats. J. Comp. Neurol. 260: 299-311.

Wise, S. P., and E. G. Jones (1977) Cells of origin and terminal distribution of descending projections of the rat somatic sensory cortex. J. Comp. Neurol. 175: 129-158. 